\title{
Development of the Methodology and U.S. Normalization Database for Life Cycle Impact Assessment and Sustainability Metrics
}

\author{
Jane Bare* \\ U.S. Environmental Protection Agency \\ 26 W. Martin Luther King Drive \\ Cincinnati, OH 45268 \\ T: (513) 569-7513, F: (513) 569-7111, E-mail: bare.jane@epa.gov
}

\section{Thomas Gloria}

Five Winds International

\section{Gregory Norris}

Sylvatica, Harvard School of Public Health

*Denotes corresponding author

\begin{abstract}
Normalization is an optional step within Life Cycle Impact Assessment (LCIA) that may be used to assist in the interpretation of life cycle inventory data as well as, life cycle impact assessment results. Normalization transforms the magnitude of LCI and LCIA results into relative contribution by substance and life cycle impact category. Normalization thus can significantly influence LCA-based decisions when tradeoffs exist. The U.S. Environmental Protection Agency (USEPA) has developed a normalization database based on the spatial scale of the U.S. 48 continental states, Hawaii, Alaska, the District of Columbia and Puerto Rico with a one-year reference time frame. Data within the normalization database were compiled based on the impact methodologies and lists of stressors used in TRACI - the U.S. EPA's Tool for the Reduction and Assessment of Chemical and other environmental Impacts (Bare et al. 2003). The new normalization database published within this article may be used for LCIA case studies within the U.S., and can be used to assist in the further development of a global normalization database. The underlying data analyzed for the development of this database are included within this paper to allow the development of normalization data consistent with other impact assessment methodologies as well.
\end{abstract}




\section{List of Tables and Figures}

Table 1. Acidification Substances with U.S. Annual Emissions Estimates

Figure 1. Percent Contribution by Substance to the Normalized value for Acidification

Table 2. 1999 U.S. Normalized values by Substance for Acidification

Table 3. 1999 U.S. Annual Air Emissions Estimates for Ecotoxicity Substances whose Normalized Contribution Represent $>\mathbf{0 . 0 1 \%}$ of the Total Normalized Value.

Table 4. 1999 U.S. Annual Water Emissions Estimates for Ecotoxicity Substances whose Normalized Contribution Represent $>\mathbf{0 . 0 1 \%}$ of the Total Normalized Value.

Figure 2. \% Contribution by Substance to the Air Normalized value for Ecotoxicity. Substances representing less than $1 \%$ were included within the "Sum of All Others."

Figure 3. \% Contribution by Substance to the Water Normalized value for Ecotoxicity. Substances representing less than $1 \%$ were included within the "Sum of All Others."

Table 5. 1999 U.S. Normalized values by Substance for Ecotoxicity Air Estimates for all substance with $>0.01 \%$ contribution to total normalized value.

Table 6. 1999 U.S. Normalized values by Substance for Ecotoxicity Water Estimates for all substance with $>0.01 \%$ contribution to total normalized value.

Table 7. U.S. Annual Emissions Estimates of Eutrophication Substances Released to the Air

Table 8. U.S. Annual Emissions Estimates of Eutrophication Substances Released to the Water

Figure 4. Percent Contribution by Substance to the Normalized value for Eutrophication effects due to air emissions

Figure 5. Percent Contribution by Substance to the Normalized value for Eutrophication effects due to water emissions

Table 9. 1999 U.S. Normalized values by Substance for Eutrophication Air

Table 10. 1999 U.S. Normalized values by Substance for Eutrophication Water

Table 11. Global Warming Substances with U.S. Annual Emission Estimates

Figure 6. Percent Contribution by Substance to the Normalized value for Global Warming

Table 12. 1999 U.S. Normalized values by Substance for Global Warming

Table 13. 1999 U.S. Annual Air Emissions Estimates for Human Health Cancer Substances whose Normalized Contribution Represent $>\mathbf{0 . 0 1 \%}$ of the Total Normalized Value.

Table 14. 1999 U.S. Annual Water Emissions Estimates for Human Health Cancer Substances whose Normalized Contribution Represent $>0.01 \%$ of the Total Normalized Value.

Figure 7. \% Contribution by Substance to the Air Normalized value for Human health cancer. Substances representing less than $1 \%$ were included within the "Sum of All Others."

Figure 8. \% Contribution by Substance to the Water Normalized value for Human Health Cancer. Substances representing less than $1 \%$ were included within the "Sum of All Others."

Table 15. 1999 U.S. Normalized values by Substance for Human Health Cancer Air Estimates for all substance with $>0.01 \%$ contribution to total normalized value

Table 16. 1999 U.S. Normalized values by Substance for Human Health Cancer Water Estimates for all substance with $>\mathbf{0 . 0 1 \%}$ contribution to total normalized value 
Table 17. 1999 U.S. Annual Air Emissions Estimates for Human Health Noncancer Substances whose Normalized Contribution Represent $>0.01 \%$ of the Total Normalized Value

Table 18. 1999 U.S. Annual Water Emissions Estimates for Human Health Noncancer Substances whose Normalized Contribution Represent $\mathbf{> 0 . 0 1 \%}$ of the Total Normalized Value

Figure 9. \% Contribution by Substance to the Air Normalized value for Human health Noncancer. Substances representing less than $1 \%$ were included within the "Sum of All Others."

Figure 10. \% Contribution by Substance to the Water Normalized value for Human health Noncancer. Substances representing less than $1 \%$ were included within the "Sum of All Others."

Table 19.1999 U.S. Normalized values by Substance for Human Health Noncancer Air Estimates for all substance with $>\mathbf{0 . 0 1 \%}$ contribution to total normalized value.

Table 20. 1999 U.S. Normalized values by Substance for Human Health Noncancer Water Estimates for all substance with $\mathbf{> 0 . 0 1 \%}$ contribution to total normalized value.

Table 21. Human Health Criteria Substances with U.S. Annual Emissions Estimates

Figure 11. Percent Contribution by Substance to the Normalized value for Human Health Criteria

Table 22. 1999 U.S. Normalized values by Substance for Human Health Criteria

Table 23. Ozone Depleting Substances with U.S. Annual Emissions Estimates

Figure 12. Percent Contribution by Substance to the Normalized value for Ozone Depleting Substances.

Table 24. 1999 U.S. Normalized values by Substance for Ozone Depletion Potential

Table 25. Photochemical Smog Formation Pollutants with U.S. Annual Emissions Estimates

Figure 13. Percent Contribution by Substance to the Normalized value for Photochemical Smog Formation.

Table 26. 1999 U.S. Normalized values by Substance for Photochemical Smog Formation

Table 27. Fossil Fuels with U.S. Annual Consumption Estimates

Figure 14. Percent Contribution by Fuel to the Normalized value for Fossil Fuel Use

Table 28. 1999 U.S. Normalized values by Substance for Fossil Fuel Use. 
ACIDIFICATION

\begin{tabular}{|l|c|c|}
\hline \multicolumn{1}{|c|}{ Substance Name } & $\begin{array}{c}\text { U.S. Annual } \\
\text { Emissions Estimate } \\
\text { (kg/yr) }\end{array}$ & Reference \\
\hline Nitrogen Oxides $\left(\mathrm{NO}_{x}\right)$ & $2.07 \mathrm{E}+10$ & $\mathrm{NEI}$ \\
\hline Sulfur Dioxide $\left(\mathrm{SO}_{2}\right)$ & $1.59 \mathrm{E}+10$ & $\mathrm{NEI}$ \\
\hline Ammonia & $4.41 \mathrm{E}+09$ & $\mathrm{NEI}$ \\
\hline Hydrochloric Acid & $3.04 \mathrm{E}+08$ & TRI \\
\hline Hydrofluoric Acid & $3.31 \mathrm{E}+07$ & TRI \\
\hline
\end{tabular}

Table 1. Acidification Substances with U.S. Annual Emissions Estimates

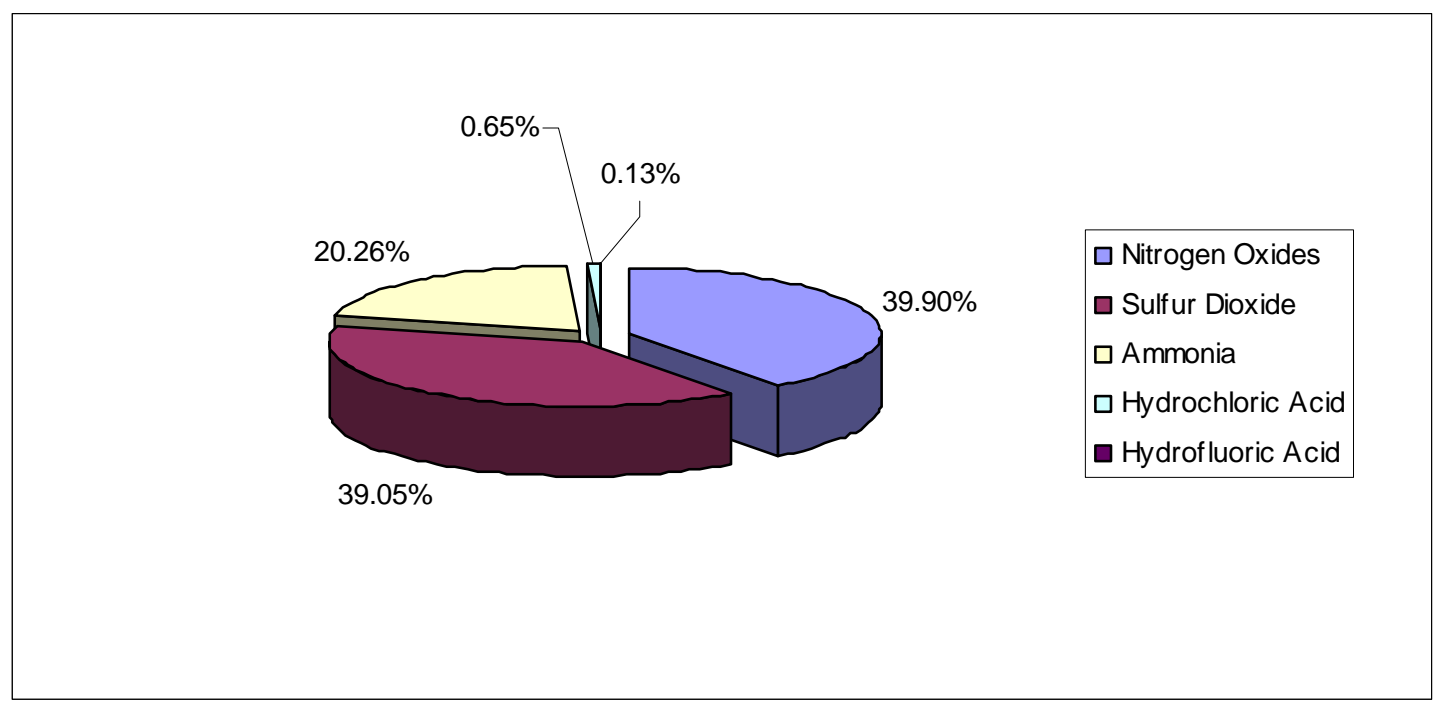

Figure 1. Percent Contribution by Substance to the Normalized value for Acidification

\begin{tabular}{|l|c|c|c|}
\hline \multicolumn{1}{|c|}{ Substance Name } & $\begin{array}{c}\text { U.S. Normalized values } \\
\text { (kg H+ eq/year) }\end{array}$ & $\begin{array}{c}\text { U.S. Normalized values } \\
\text { (kg H+ eq/year per capita) }\end{array}$ & $\begin{array}{c}\text { Percent } \\
\text { Contribution to } \\
\text { Total }\end{array}$ \\
\hline Ammonia & $8.29 \mathrm{E}+11$ & $2.97 \mathrm{E}+03$ & $39.90 \%$ \\
\hline Sulfur Dioxide & $8.11 \mathrm{E}+11$ & $2.91 \mathrm{E}+03$ & $39.05 \%$ \\
\hline Nitrogen Oxides & $4.21 \mathrm{E}+11$ & $1.51 \mathrm{E}+03$ & $20.26 \%$ \\
\hline Hydrochloric Acid & $1.36 \mathrm{E}+10$ & $4.87 \mathrm{E}+01$ & $0.65 \%$ \\
\hline Hydrofluoric Acid & $2.69 \mathrm{E}+09$ & $9.64 \mathrm{E}+00$ & $0.13 \%$ \\
\hline Total & $\mathbf{2 . 0 8 E + 1 2}$ & $\mathbf{7 . 4 4 E + 0 3}$ & $\mathbf{1 0 0 . 0 \%}$ \\
\hline
\end{tabular}

Table 2. 1999 U.S. Normalized values by Substance for Acidification 


\section{ECOTOXICITY}

\begin{tabular}{|l|c|c|c|}
\hline \multicolumn{1}{|c|}{ Chemical Name } & $\begin{array}{c}\text { TRI Annual Air } \\
\text { Emissions } \\
\text { Esimate } \mathbf{( k g / y r )}\end{array}$ & $\begin{array}{c}\text { NEI Annual Air } \\
\text { Emissions } \\
\text { Estimate } \mathbf{( k g / y r )}\end{array}$ & $\begin{array}{c}\text { Data Source } \\
\text { Selected (TRI or } \\
\text { NEI or None) }\end{array}$ \\
\hline COPPER & $7.04 \mathrm{E}+05$ & $0.00 \mathrm{E}+00$ & $\mathrm{TRI}$ \\
\hline ZINC & $6.39 \mathrm{E}+05$ & $0.00 \mathrm{E}+00$ & $\mathrm{TRI}$ \\
\hline NICKEL & $1.27 \mathrm{E}+05$ & $0.00 \mathrm{E}+00$ & $\mathrm{TRI}$ \\
\hline CHROMIUM & $1.34 \mathrm{E}+05$ & $0.00 \mathrm{E}+00$ & $\mathrm{TRI}$ \\
\hline DIAZINON & $1.20 \mathrm{E}+04$ & $0.00 \mathrm{E}+00$ & $\mathrm{TRI}$ \\
\hline SELENIUM & $3.17 \mathrm{E}+04$ & $0.00 \mathrm{E}+00$ & $\mathrm{TRI}$ \\
\hline BIFENTHRIN & $2.48 \mathrm{E}+02$ & $0.00 \mathrm{E}+00$ & $\mathrm{TRI}$ \\
\hline
\end{tabular}

Table 3. 1999 U.S. Annual Air Emissions Estimates for Ecotoxicity Substances whose Normalized Contribution Represent $>\mathbf{0 . 0 1 \%}$ of the Total Normalized Value.

\begin{tabular}{|l|c|c|}
\hline \multicolumn{1}{|c|}{ Substance Name } & $\begin{array}{c}\text { TRI Annual Water } \\
\text { Emissions Esimate } \\
(\mathbf{k g} / \mathbf{y r})\end{array}$ & $\begin{array}{c}\text { Reference } \\
\text { Number }\end{array}$ \\
\hline COPPER & $1.66 \mathrm{E}+04$ & TRI \\
\hline NICKEL & $1.21 \mathrm{E}+04$ & TRI \\
\hline ZINC & $1.06 \mathrm{E}+04$ & TRI \\
\hline CHROMIUM & $1.45 \mathrm{E}+04$ & TRI \\
\hline MERCURY & $6.03 \mathrm{E}+01$ & TRI \\
\hline THALLIUM & $2.72 \mathrm{E}+02$ & TRI \\
\hline SELENIUM & $1.29 \mathrm{E}+02$ & TRI \\
\hline
\end{tabular}

Table 4. 1999 U.S. Annual Water Emissions Estimates for Ecotoxicity Substances whose Normalized Contribution Represent $>\mathbf{0 . 0 1 \%}$ of the Total Normalized Value. 


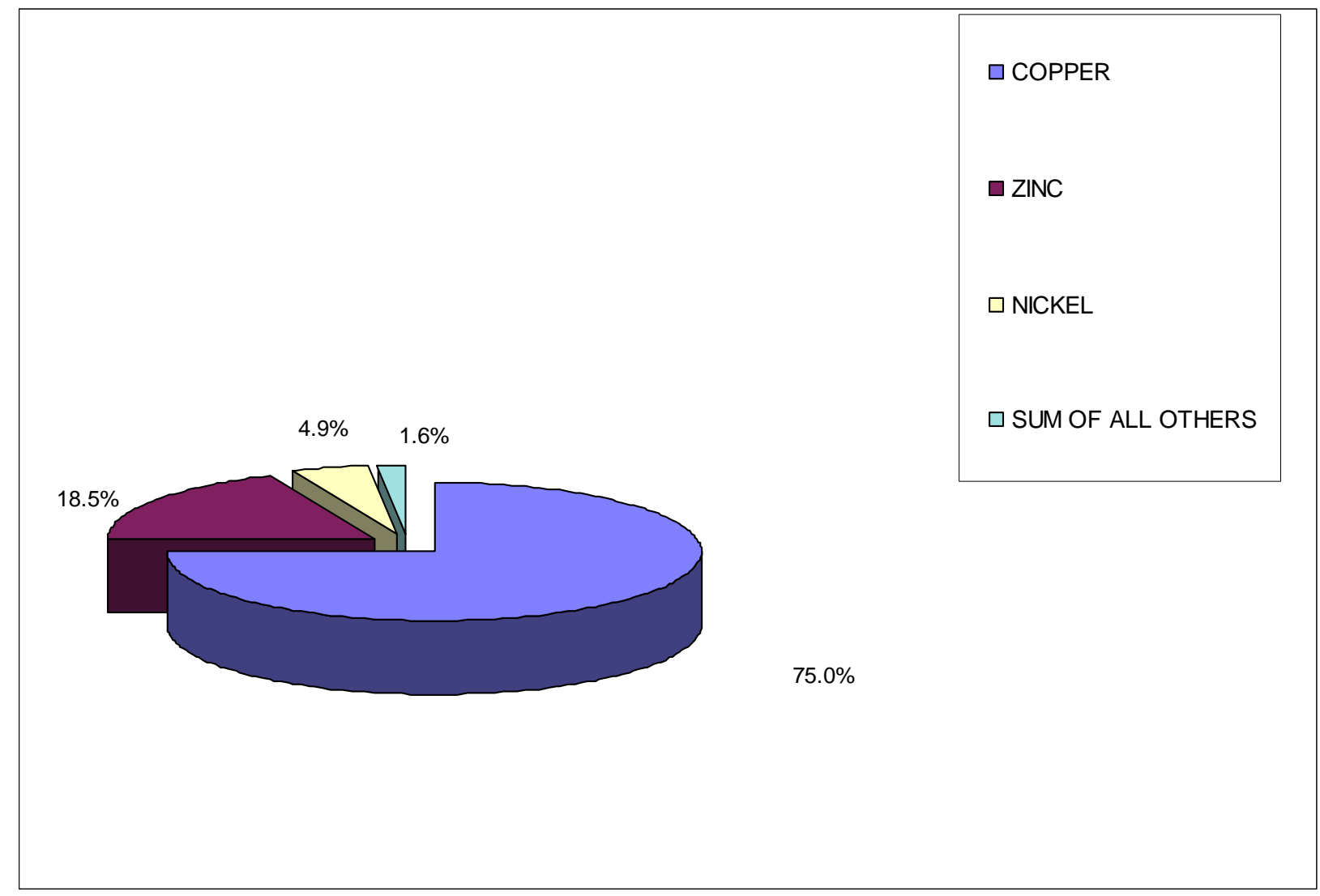

Figure 2. \% Contribution by Substance to the Air Normalized value for Ecotoxicity. Substances representing less than $1 \%$ were included within the "Sum of All Others."

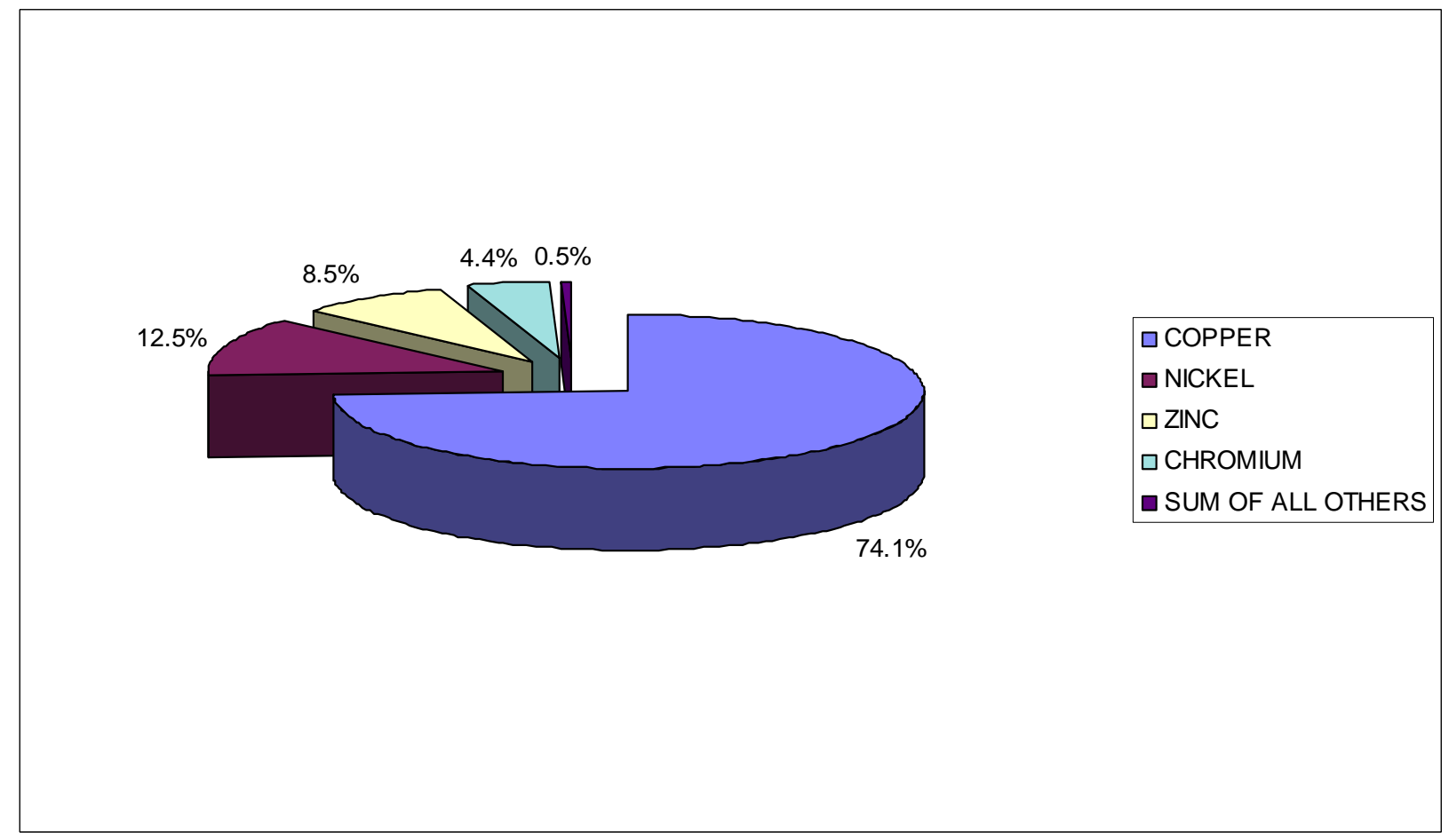


Figure 3. \% Contribution by Substance to the Air Normalized value for Ecotoxicity. Substances representing less than $1 \%$ were included within the "Sum of All Others."

\begin{tabular}{|l|c|c|c|}
\hline \multicolumn{1}{|c|}{ Chemical Name } & $\begin{array}{c}\text { U.S. Normalized Air Values } \\
(2,4-\mathrm{D} \text { eq/yr) }\end{array}$ & $\begin{array}{c}\text { U.S. Normalized Air } \\
\text { Value Per Capita (2,4- } \\
\text { D eq/yr per capita })\end{array}$ & $\begin{array}{c}\text { Percentage } \\
\text { Contribution to Total }\end{array}$ \\
\hline COPPER & $1.53 \mathrm{E}+10$ & $5.47 \mathrm{E}+01$ & $75.0 \%$ \\
\hline ZINC & $3.76 \mathrm{E}+09$ & $1.35 \mathrm{E}+01$ & $18.5 \%$ \\
\hline NICKEL & $9.94 \mathrm{E}+08$ & $3.56 \mathrm{E}+00$ & $4.9 \%$ \\
\hline CHROMIUM & $1.40 \mathrm{E}+08$ & $5.02 \mathrm{E}-01$ & $0.7 \%$ \\
\hline DIAZINON & $1.01 \mathrm{E}+08$ & $3.60 \mathrm{E}-01$ & $0.5 \%$ \\
\hline SELENIUM & $4.84 \mathrm{E}+07$ & $1.73 \mathrm{E}-01$ & $0.2 \%$ \\
\hline BIFENTHRIN & $2.22 \mathrm{E}+07$ & $7.96 \mathrm{E}-02$ & $0.1 \%$ \\
\hline SUM OF ALL OTHERS & $2.13 \mathrm{E}+07$ & $7.65 \mathrm{E}-02$ & $0.1 \%$ \\
\hline TOTAL & $2.03 \mathrm{E}+10$ & $7.29 \mathrm{E}+01$ & $100.0 \%$ \\
\hline
\end{tabular}

Table 5. 1999 U.S. Normalized values by Substance for Ecotoxicity Water Estimates for all substance with $>0.01 \%$ contribution to total normalized value.

\begin{tabular}{|l|c|c|c|}
\hline \multicolumn{1}{|c|}{ Chemical Name } & $\begin{array}{c}\text { U.S. Normalized } \\
\text { Water Values (2,4- } \\
\text { D eq/yr) }\end{array}$ & $\begin{array}{c}\text { U.S. Normalized } \\
\text { Water Value Per } \\
\text { Capita (2,4-D } \\
\text { eq/yr per capita) }\end{array}$ & $\begin{array}{c}\text { Percentage } \\
\text { Contribution to } \\
\text { Total }\end{array}$ \\
\hline COPPER & $1.91 \mathrm{E}+08$ & $6.85 \mathrm{E}-01$ & $74.1 \%$ \\
\hline NICKEL & $3.23 \mathrm{E}+07$ & $1.16 \mathrm{E}-01$ & $12.5 \%$ \\
\hline ZINC & $2.18 \mathrm{E}+07$ & $7.82 \mathrm{E}-02$ & $8.5 \%$ \\
\hline CHROMIUM & $1.13 \mathrm{E}+07$ & $4.04 \mathrm{E}-02$ & $4.4 \%$ \\
\hline MERCURY & $1.88 \mathrm{E}+05$ & $6.73 \mathrm{E}-04$ & $0.1 \%$ \\
\hline THALLIUM & $1.66 \mathrm{E}+05$ & $5.96 \mathrm{E}-04$ & $0.1 \%$ \\
\hline SELENIUM & $1.39 \mathrm{E}+05$ & $4.98 \mathrm{E}-04$ & $0.1 \%$ \\
\hline SUM OF ALL OTHERS & $8.33 \mathrm{E}+05$ & $2.98 \mathrm{E}-03$ & $0.3 \%$ \\
\hline TOTAL & $2.58 \mathrm{E}+08$ & $9.24 \mathrm{E}-01$ & $100.0 \%$ \\
\hline
\end{tabular}

Table 6. 1999 U.S. Normalized values by Substance for Ecotoxicity Water Estimates for all substance with $>0.01 \%$ contribution to total normalized value.

\section{EUTROPHICATION}

\begin{tabular}{|l|c|c|c|}
\hline \multicolumn{1}{|c|}{ Substance Name } & $\begin{array}{c}\text { U.S. Annual Air } \\
\text { Emissions Estimate }\end{array}$ & Unit & Reference \\
\hline Nitrogen Oxides $\left(\mathrm{NO}_{\mathrm{x}}\right)$ & $2.06 \mathrm{E}+10$ & $\mathrm{~kg} / \mathrm{year}$ & $\mathrm{NEI}$ \\
\hline Ammonia & $4.40 \mathrm{E}+09$ & $\mathrm{~kg} / \mathrm{year}$ & $\mathrm{NEI}$ \\
\hline
\end{tabular}

Table 7. U.S. Annual Emissions Estimates of Eutrophication Substances Released to the Air

\begin{tabular}{|l|c|c|c|}
\hline \multicolumn{1}{|c|}{ Substance Name } & $\begin{array}{c}\text { U.S. Annual Water } \\
\text { Emissions Estimate }\end{array}$ & Unit & Reference \\
\hline Phosphorus & $4.20 \mathrm{E}+08$ & $\mathrm{~kg} / \mathrm{year}$ & USDA, 2000 \\
\hline Nitrogen & $6.67 \mathrm{E}+08$ & $\mathrm{~kg} / \mathrm{year}$ & USDA, 2000 \\
\hline
\end{tabular}


Table 8. U.S. Annual Emissions Estimates of Eutrophication Substances Released to the Water

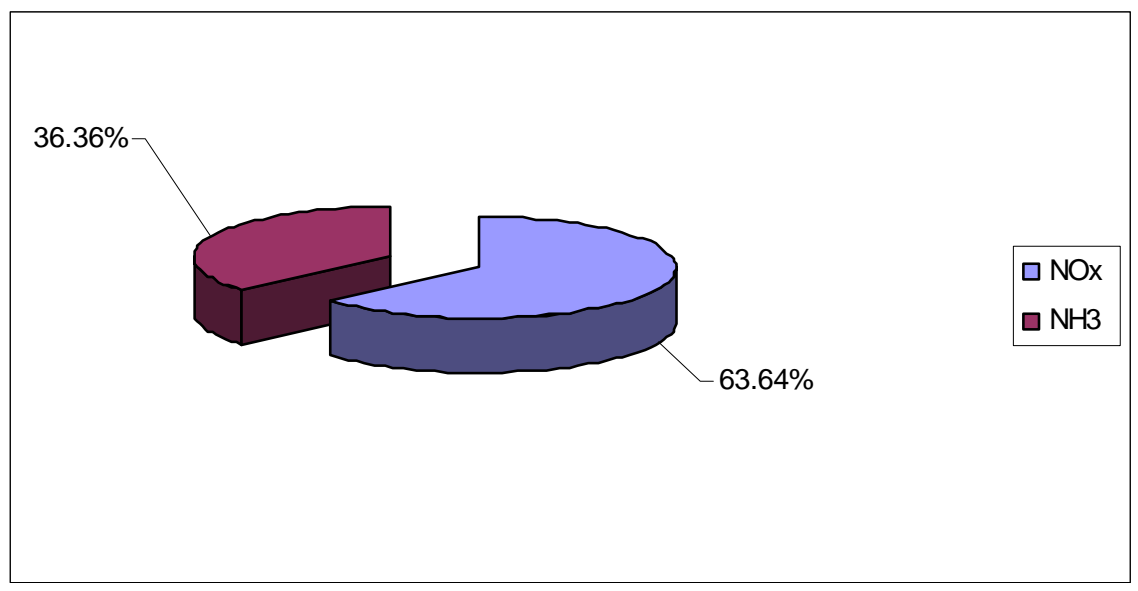

Figure 4. Percent Contribution by Substance to the Normalized value for Eutrophication effects due to air emissions

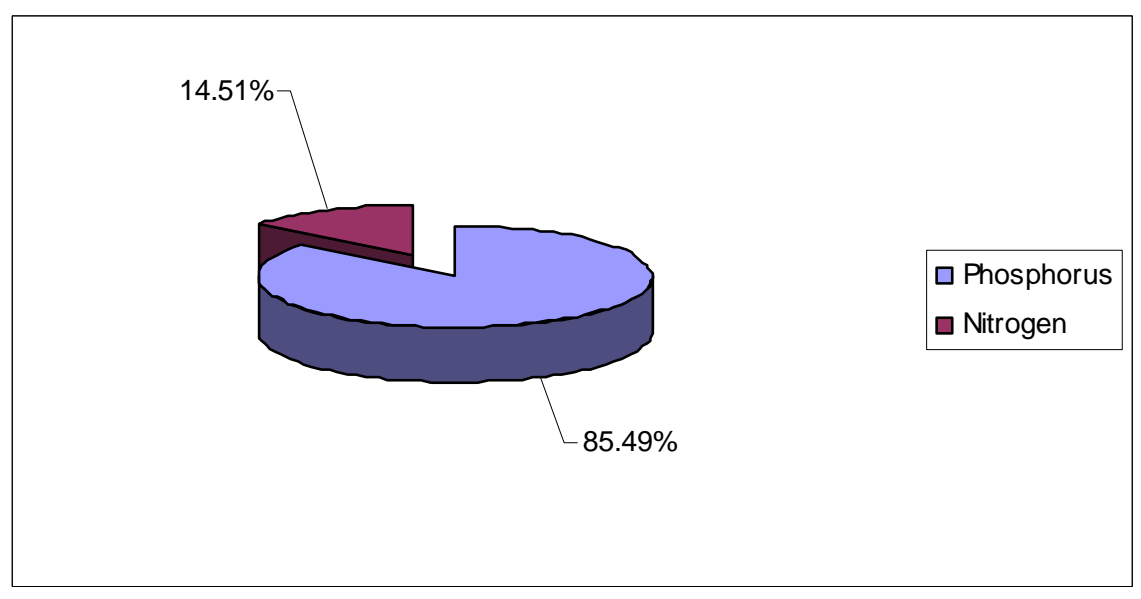

Figure 5. Percent Contribution by Substance to the Normalized value for Eutrophication effects due to water emissions

\begin{tabular}{|l|c|c|c|}
\hline \multicolumn{1}{|c|}{ Substance Name } & $\begin{array}{c}\text { U.S. Normalized air value } \\
\text { (kg Nitrogen eq/kg } \\
\text { emission/year) }\end{array}$ & $\begin{array}{c}\text { U.S. Normalized air value } \\
\text { (kg Nitrogen-eq/ kg } \\
\text { emission/year per capita) }\end{array}$ & $\begin{array}{c}\text { Contribution to } \\
\text { Total } \\
\text { (in \%) }\end{array}$ \\
\hline $\begin{array}{l}\text { Nitrogen Oxides } \\
(\mathrm{NO})\end{array}$ & $9.14 \mathrm{E}+08$ & $3.28 \mathrm{E}+00$ & $63.64 \%$ \\
\hline Ammonia & $5.22 \mathrm{E}+08$ & $1.87 \mathrm{E}+00$ & $36.36 \%$ \\
\hline Total & $\mathbf{1 . 4 4 E + 0 9}$ & $\mathbf{5 . 1 5 E + 0 0}$ & $\mathbf{1 0 0 . 0 0 \%}$ \\
\hline
\end{tabular}

Table 9. 1999 U.S. Normalized values by Substance for Eutrophication Air

\begin{tabular}{|c|c|c|c|}
\hline Substance Name & $\begin{array}{c}\text { U.S. Normalized water } \\
\text { value } \\
\text { (kg Nitrogen eq/kg } \\
\text { emission/year) }\end{array}$ & $\begin{array}{c}\text { U.S. Normalized water } \\
\text { value } \\
\text { (kg Nitrogen-eq/ } \mathbf{k g} \\
\text { emission/year per capita) }\end{array}$ & $\begin{array}{c}\text { Contribution to } \\
\text { Total } \\
\text { (in \%) }\end{array}$ \\
\hline
\end{tabular}




\begin{tabular}{|l|c|c|c|}
\hline Phosphorus & $3.06 \mathrm{E}+09$ & $1.10 \mathrm{E}+01$ & $85.49 \%$ \\
\hline Nitrogen & $5.20 \mathrm{E}+08$ & $1.86 \mathrm{E}+00$ & $14.51 \%$ \\
\hline Total & $\mathbf{3 . 5 8 E}+09$ & $\mathbf{1 . 2 8 E}+\mathbf{0 1}$ & $\mathbf{1 0 0 . 0 0 \%}$ \\
\hline
\end{tabular}

Table 10. 1999 U.S. Normalized values by Substance for Eutrophication Water

GLOBAL WARMING

\begin{tabular}{|c|c|c|c|}
\hline Substance Name & $\begin{array}{l}\text { U.S. Annual } \\
\text { Emissions } \\
\text { Estimate }\end{array}$ & Unit & Reference \\
\hline Carbon Dioxide & $5.68 \mathrm{E}+12$ & kg/year & USEPA, 2004b \\
\hline Methane & $2.92 \mathrm{E}+10$ & $\mathrm{~kg} /$ year & USEPA, 2004b \\
\hline Nitrous Oxide & $1.38 \mathrm{E}+09$ & $\mathrm{~kg} /$ year & USEPA, 2004b \\
\hline HFC-134A & $3.25 \mathrm{E}+07$ & $\mathrm{~kg} /$ year & USEPA, 2004b, 2004c \\
\hline HFC-23 & $2.64 \mathrm{E}+06$ & $\mathrm{~kg} /$ year & $\begin{array}{c}\text { USEPA, 2004b, 2004c, } \\
2004 \mathrm{e}\end{array}$ \\
\hline Sulfur Hexafluoride & $9.92 \mathrm{E}+05$ & $\mathrm{~kg} /$ year & $\begin{array}{c}\text { USEPA, 2004b, 2000d, } \\
2004 \mathrm{e}\end{array}$ \\
\hline HFC-125 & $3.57 \mathrm{E}+06$ & $\mathrm{~kg} / \mathrm{year}$ & USEPA, 2004b, 2004c \\
\hline $\begin{array}{l}\text { Carbon Tetrafluoride } \\
\text { (CF4) }\end{array}$ & $1.51 \mathrm{E}+06$ & $\mathrm{~kg} /$ year & USEPA, 2004b \\
\hline HFC-143A & $1.74 \mathrm{E}+06$ & $\mathrm{~kg} /$ year & USEPA, 2004b, 2004c \\
\hline $\begin{array}{l}\text { Other HFCs (HFC-152a, } \\
\text { HFC-227ea, HFC-245fa, } \\
\text { HFC-4310mee) }\end{array}$ & $4.65 E+06$ & $\mathrm{~kg} /$ year & USEPA, 2004b, 2004c \\
\hline Perfluoroethane (C2F6) & $5.00 \mathrm{E}+05$ & $\mathrm{~kg} /$ year & $\begin{array}{c}\text { USEPA, 2004b, 2000d, } \\
2004 \mathrm{e}\end{array}$ \\
\hline HFC-236FA & $1.43 \mathrm{E}+05$ & $\mathrm{~kg} /$ year & $\begin{array}{l}\text { USEPA, 2004b, 2004c, } \\
2000 d, 2004 \mathrm{e}\end{array}$ \\
\hline HFC-32 & $4.62 \mathrm{E}+05$ & $\mathrm{~kg} /$ year & USEPA, 2004b, 2004c \\
\hline
\end{tabular}


Table 11. Global Warming Substances with U.S. Annual Emission Estimates

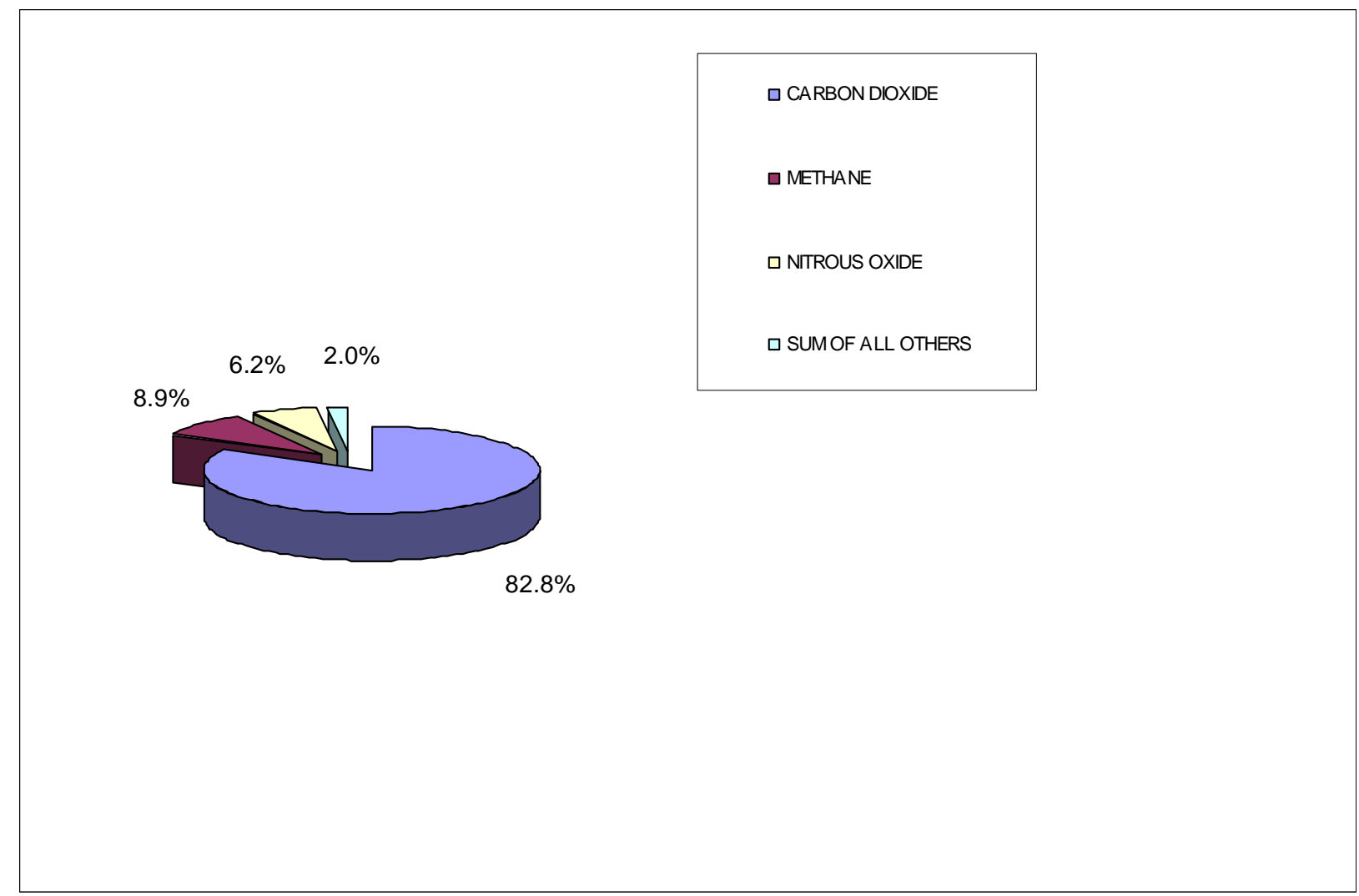

Figure 6. Percent Contribution by Substance to the Normalized value for Global Warming

\begin{tabular}{|l|c|c|c|}
\hline \multicolumn{1}{|c|}{ Substance Name } & $\begin{array}{c}\text { U.S. Normalized } \\
\text { Values (CO2 eq/yr) }\end{array}$ & $\begin{array}{c}\text { Per Capita } \\
\text { Normalized Values } \\
\text { (CO2 eq/yr per } \\
\text { capita) }\end{array}$ & $\begin{array}{c}\text { Percent } \\
\text { Contribution to } \\
\text { Total }\end{array}$ \\
\hline CARBON DIOXIDE & $5.68 \mathrm{E}+12$ & $2.03 \mathrm{E}+04$ & $\mathbf{8 2 . 8 \%}$ \\
\hline METHANE & $6.13 \mathrm{E}+11$ & $2.20 \mathrm{E}+03$ & $8.9 \%$ \\
\hline NITROUS OXIDE & $4.28 \mathrm{E}+11$ & $1.53 \mathrm{E}+03$ & $6.2 \%$ \\
\hline HFC-134A & $4.22 \mathrm{E}+10$ & $1.51 \mathrm{E}+02$ & $0.6 \%$ \\
\hline HFC-23 & $3.09 \mathrm{E}+10$ & $1.11 \mathrm{E}+02$ & $0.5 \%$ \\
\hline SULFUR HEXAFLUORIDE & $2.37 \mathrm{E}+10$ & $\mathbf{8 . 4 9 E + 0 1}$ & $0.3 \%$ \\
\hline HFC-125 & $1.00 \mathrm{E}+10$ & $3.58 \mathrm{E}+01$ & $0.1 \%$ \\
\hline CARBON TETRAFLUORIDE (CF4) & $9.80 \mathrm{E}+09$ & $3.51 \mathrm{E}+01$ & $0.1 \%$ \\
\hline HFC-143A & $6.60 \mathrm{E}+09$ & $2.36 \mathrm{E}+01$ & $0.1 \%$ \\
\hline Other HFCs & $5.70 \mathrm{E}+09$ & $2.04 \mathrm{E}+01$ & $0.1 \%$ \\
\hline PERFLUOROETHANE (C2F6) & $4.60 \mathrm{E}+09$ & $1.65 \mathrm{E}+01$ & $0.1 \%$ \\
\hline HFC-236FA & $9.00 \mathrm{E}+08$ & $3.22 \mathrm{E}+00$ & $0.0 \%$ \\
\hline HFC-32 & $3.00 \mathrm{E}+08$ & $1.07 \mathrm{E}+00$ & $0.0 \%$ \\
\hline TOTAL & $6.85 \mathrm{E}+12$ & $2.45 \mathrm{E}+04$ & $100.0 \%$ \\
\hline
\end{tabular}

Table 12. 1999 U.S. Normalized values by Substance for Global Warming 
HUMAN HEALTH CANCER

\begin{tabular}{|c|c|c|c|}
\hline Substance Name & $\begin{array}{l}\text { TRI Annual Air } \\
\text { Emissions } \\
\text { Esimate (kg/yr) }\end{array}$ & $\begin{array}{c}\text { NEI Annual } \\
\text { Emissions } \\
\text { Estimate (kg/yr) }\end{array}$ & $\begin{array}{l}\text { Data Source } \\
\text { Selected (TRI or } \\
\text { NEI or None) }\end{array}$ \\
\hline ARSENIC & $2.9 \mathrm{E}+03$ & $0.0 \mathrm{E}+00$ & TRI \\
\hline CHROMIUM & $1.3 \mathrm{E}+05$ & $0.0 \mathrm{E}+00$ & $\overline{T R I}$ \\
\hline LEAD & $1.4 \mathrm{E}+05$ & $0.0 \mathrm{E}+00$ & TRI \\
\hline ACRYLAMIDE & $1.1 \mathrm{E}+04$ & $8.0 \mathrm{E}+00$ & TRI \\
\hline BENZENE & $3.6 \mathrm{E}+06$ & $1.6 \mathrm{E}+05$ & $\mathrm{TRI}$ \\
\hline METHYL IODIDE & $3.1 \mathrm{E}+04$ & $2.6 \mathrm{E}+01$ & $\mathrm{TRI}$ \\
\hline ETHYLENE OXIDE & $2.2 \mathrm{E}+05$ & $5.6 \mathrm{E}+02$ & $\mathrm{TRI}$ \\
\hline DICHLOROMETHANE & $1.6 \mathrm{E}+07$ & $3.8 \mathrm{E}+04$ & $\mathrm{TRI}$ \\
\hline CHLOROFORM & $2.4 \mathrm{E}+06$ & $6.9 \mathrm{E}+03$ & TRI \\
\hline TETRACHLOROETHYLENE & $1.8 \mathrm{E}+06$ & $2.1 \mathrm{E}+04$ & $\overline{T R I}$ \\
\hline ACRYLONITRILE & $4.5 \mathrm{E}+05$ & $5.9 \mathrm{E}+02$ & $\overline{T R I}$ \\
\hline ALLYL TRICHLORIDE & $6.3 \mathrm{E}+03$ & $0.0 \mathrm{E}+00$ & $\overline{\mathrm{TRI}}$ \\
\hline CARBON TETRACHLORIDE & $1.1 \mathrm{E}+05$ & $8.8 \mathrm{E}+02$ & $\overline{\mathrm{TRI}}$ \\
\hline HYDROQUINONE & $2.7 \mathrm{E}+04$ & $3.7 \mathrm{E}+01$ & TRI \\
\hline DIMETHYL SULFATE & $4.5 \mathrm{E}+03$ & $4.1 \mathrm{E}+00$ & TRI \\
\hline VINYL CHLORIDE & $3.9 \mathrm{E}+05$ & $6.1 \mathrm{E}+02$ & TRI \\
\hline 1,2-DICHLOROETHANE & $2.5 \mathrm{E}+05$ & $4.2 \mathrm{E}+02$ & TRI \\
\hline 1,3-BUTADIENE & $9.4 \mathrm{E}+05$ & $2.6 \mathrm{E}+04$ & TRI \\
\hline METHYL CHLORIDE & $1.3 \mathrm{E}+06$ & $1.6 \mathrm{E}+04$ & $\overline{T R I}$ \\
\hline ATRAZINE & $9.5 \mathrm{E}+03$ & $0.0 \mathrm{E}+00$ & TRI \\
\hline BENZIDINE & $5.9 \mathrm{E}+00$ & $3.1 \mathrm{E}-01$ & $\overline{T R I}$ \\
\hline TRICHLOROETHYLENE & $4.8 \mathrm{E}+06$ & $1.1 \mathrm{E}+04$ & $\overline{T R I}$ \\
\hline BENZOIC TRICHLORIDE & $9.9 \mathrm{E}+02$ & $1.3 \mathrm{E}+00$ & $\overline{T R I}$ \\
\hline NICKEL & $1.3 \mathrm{E}+05$ & $0.0 \mathrm{E}+00$ & $\overline{T R I}$ \\
\hline 4,4'-DIAMINO DITAN & $4.2 \mathrm{E}+03$ & $8.1 \mathrm{E}+00$ & TRI \\
\hline 1,1,2-TRICHLOROETHANE & $9.0 \mathrm{E}+04$ & $3.0 \mathrm{E}+02$ & $\mathrm{TRI}$ \\
\hline 1,2-DICHLOROPROPANE & $1.1 \mathrm{E}+05$ & $7.1 \mathrm{E}+01$ & $\mathrm{TRI}$ \\
\hline BENZO(B)PYRIDINE & $5.4 \mathrm{E}+03$ & $7.2 \mathrm{E}+00$ & $\mathrm{TRI}$ \\
\hline PROPYLENE OXIDE & $3.2 \mathrm{E}+05$ & $2.8 \mathrm{E}+02$ & TRI \\
\hline 1,4-DICHLOROBENZENE & $8.1 \mathrm{E}+04$ & $5.8 \mathrm{E}+03$ & TRI \\
\hline 1-CHLORO-2,3-EPOXYPROPANE & $7.1 \mathrm{E}+04$ & $5.2 \mathrm{E}+01$ & TRI \\
\hline SIMAZINE & $1.8 \mathrm{E}+03$ & $0.0 \mathrm{E}+00$ & TRI \\
\hline 2-NITROPROPANE & $9.5 \mathrm{E}+03$ & $7.4 \mathrm{E}+00$ & TRI \\
\hline CADMIUM & $1.9 \mathrm{E}+03$ & $0.0 \mathrm{E}+00$ & TRI \\
\hline HEXACHLOROETHANE & $1.9 \mathrm{E}+04$ & $2.2 \mathrm{E}+01$ & $\overline{T R I}$ \\
\hline NITROGLYCERIN & $7.0 \mathrm{E}+03$ & $0.0 \mathrm{E}+00$ & TRI \\
\hline 1,2-DIBROMOETHANE & $5.5 \mathrm{E}+03$ & $6.1 \mathrm{E}+00$ & TRI \\
\hline DICHLOROBENZENE (MIXED ISOMERS) & $6.3 \mathrm{E}+03$ & $0.0 \mathrm{E}+00$ & TRI \\
\hline HYDRAZINE & $4.7 \mathrm{E}+03$ & $2.5 \mathrm{E}+01$ & TRI \\
\hline DICOFOL & $4.6 \mathrm{E}+02$ & $0.0 \mathrm{E}+00$ & TRI \\
\hline 1,1-DICHLOROETHYLENE & $7.2 \mathrm{E}+04$ & $5.6 \mathrm{E}+01$ & TRI \\
\hline CYANAZINE & $6.5 \mathrm{E}+01$ & $0.0 \mathrm{E}+00$ & TRI \\
\hline ACETALDEHYDE & $5.5 \mathrm{E}+06$ & $4.2 \mathrm{E}+04$ & TRI \\
\hline HEXACHLOROBENZENE & $2.6 \mathrm{E}+02$ & $2.4 \mathrm{E}+00$ & TRI \\
\hline 1,1,2,2-TETRACHLOROETHANE & $2.4 \mathrm{E}+03$ & $1.3 \mathrm{E}+02$ & TRI \\
\hline FORMALDEHYDE & $5.6 \mathrm{E}+06$ & $1.3 \mathrm{E}+05$ & $\overline{T R I}$ \\
\hline 2,4-DIAMINOTOLUENE & $3.0 \mathrm{E}+02$ & $1.9 \mathrm{E}+00$ & $\overline{\mathrm{TRI}}$ \\
\hline BIS(2-ETHYLHEXYL)PHTHALATE & $8.1 \mathrm{E}+04$ & $1.2 \mathrm{E}+02$ & TRI \\
\hline BROMODICHLOROMETHANE & $1.5 \mathrm{E}+03$ & $0.0 \mathrm{E}+00$ & TRI \\
\hline 1,1-DICHLOROETHANE & $3.7 \mathrm{E}+04$ & $1.7 \mathrm{E}+02$ & TRI \\
\hline HEXACHLORO-1,3-BUTADIENE & $2.0 \mathrm{E}+03$ & $5.5 \mathrm{E}+00$ & TRI \\
\hline BENZYL CHLORIDE & $1.2 \mathrm{E}+04$ & $4.1 \mathrm{E}+01$ & TRI \\
\hline LINURON & $4.6 \mathrm{E}+02$ & $0.0 \mathrm{E}+00$ & TRI \\
\hline PERMETHRIN & $1.2 \mathrm{E}+03$ & $0.0 \mathrm{E}+00$ & TRI \\
\hline 2,4-D [ACETIC ACID (2,4-DICHLOROPHENOXY)-] & $3.6 \mathrm{E}+03$ & $4.2 \mathrm{E}+02$ & TRI \\
\hline BIFENTHRIN & $2.5 \mathrm{E}+02$ & $0.0 \mathrm{E}+00$ & TRI \\
\hline TRIALLATE & $2.3 \mathrm{E}+02$ & $0.0 \mathrm{E}+00$ & $\overline{\mathrm{TRI}}$ \\
\hline CHLOROMETHYL METHYL ETHER & $6.2 \mathrm{E}+02$ & $8.5 \mathrm{E}+01$ & TRI \\
\hline THIOUREA & $4.5 \mathrm{E}+02$ & $0.0 \mathrm{E}+00$ & TRI \\
\hline BERYLLIUM & $3.5 \mathrm{E}+02$ & $0.0 \mathrm{E}+00$ & TRI \\
\hline
\end{tabular}


Table 13. 1999 U.S. Annual Air Emissions Estimates for Human Health Cancer Substances whose Normalized Contribution Represent $>\mathbf{0 . 0 1 \%}$ of the Total Normalized Value.

\begin{tabular}{|c|c|c|}
\hline Substance Name & $\begin{array}{c}\text { TRI Annual Water } \\
\text { Emissions Esimate } \\
\text { (kg/yr) } \\
\end{array}$ & Reference \\
\hline LEAD & $3.70 \mathrm{E}+03$ & TRI \\
\hline ALLYL TRICHLORIDE & $1.04 \mathrm{E}+03$ & $\mathrm{TRI}$ \\
\hline ARSENIC & $4.77 \mathrm{E}+02$ & TRI \\
\hline CHLOROFORM & $3.91 \mathrm{E}+04$ & TRI \\
\hline 1,3-BUTADIENE & $8.76 \mathrm{E}+02$ & TRI \\
\hline BENZENE & $1.26 \mathrm{E}+04$ & TRI \\
\hline 2-NITROPROPANE & $1.30 \mathrm{E}+02$ & $\overline{\mathrm{TRI}}$ \\
\hline 1,4-DIOXANE & $7.63 \mathrm{E}+04$ & $\overline{T R I}$ \\
\hline 1,2-DICHLOROPROPANE & $4.19 \mathrm{E}+03$ & TRI \\
\hline HYDRAZINE & $2.91 \mathrm{E}+02$ & $\overline{T R I}$ \\
\hline HEXACHLOROBENZENE & $3.63 \mathrm{E}+00$ & $\overline{T R I}$ \\
\hline ETHYLENE OXIDE & $5.25 \mathrm{E}+02$ & TRI \\
\hline PROPYLENE OXIDE & $4.92 \mathrm{E}+03$ & $\mathrm{TRI}$ \\
\hline CHLORDANE & $4.54 \mathrm{E}-01$ & $\overline{\mathrm{TRI}}$ \\
\hline 4,4'-DIAMINO DITAN & $1.93 \mathrm{E}+03$ & TRI \\
\hline 1,2-DICHLOROETHANE & $4.10 \mathrm{E}+02$ & $\overline{T R I}$ \\
\hline 1,1,2-TRICHLOROETHANE & $4.20 \mathrm{E}+02$ & TRI \\
\hline ACRYLONITRILE & $5.32 \mathrm{E}+02$ & TRI \\
\hline BIS(2-ETHYLHEXYL)PHTHALATE & $1.31 \mathrm{E}+03$ & $\overline{T R I}$ \\
\hline ACETALDEHYDE & $1.04 \mathrm{E}+05$ & $\overline{T R I}$ \\
\hline 1,4-DICHLOROBENZENE & $8.53 \mathrm{E}+02$ & $\overline{T R I}$ \\
\hline DICHLOROMETHANE & $5.48 \mathrm{E}+03$ & $\overline{T R I}$ \\
\hline TETRACHLOROETHYLENE & $8.14 \mathrm{E}+02$ & $\overline{T R I}$ \\
\hline CARBON TETRACHLORIDE & $3.86 \mathrm{E}+01$ & TRI \\
\hline 1,3-DICHLOROBENZENE & $3.67 \mathrm{E}+02$ & $\mathrm{TRI}$ \\
\hline BIS(2-CHLOROETHYL)ETHER & $1.09 \mathrm{E}+01$ & $\overline{T R I}$ \\
\hline 1,1-DICHLOROETHYLENE & $6.03 \mathrm{E}+01$ & $\mathrm{TRI}$ \\
\hline VINYL CHLORIDE & $4.67 \mathrm{E}+01$ & TRI \\
\hline ACRYLAMIDE & $1.68 \mathrm{E}+02$ & $\overline{T R I}$ \\
\hline METHYL CHLORIDE & $9.79 \mathrm{E}+02$ & $\mathrm{TRI}$ \\
\hline METHYL IODIDE & $3.63 \mathrm{E}+00$ & TRI \\
\hline BENZO(B)PYRIDINE & $1.18 \mathrm{E}+01$ & $\overline{T R I}$ \\
\hline GAMMA-HCH (LINDANE) & $2.72 \mathrm{E}+00$ & $\overline{T R I}$ \\
\hline SIMAZINE & $1.75 \mathrm{E}+02$ & $\overline{T R I}$ \\
\hline TRICHLOROETHYLENE & $4.69 \mathrm{E}+02$ & $\overline{T R I}$ \\
\hline HEPTACHLOR & $4.54 \mathrm{E}-01$ & $\overline{T R I}$ \\
\hline 1,2-DIBROMOETHANE & $5.44 \mathrm{E}+00$ & TRI \\
\hline
\end{tabular}

Table 14. 1999 U.S. Annual Water Emissions Estimates for Human Health Cancer Substances whose Normalized Contribution Represent $>0.01 \%$ of the Total Normalized Value. 


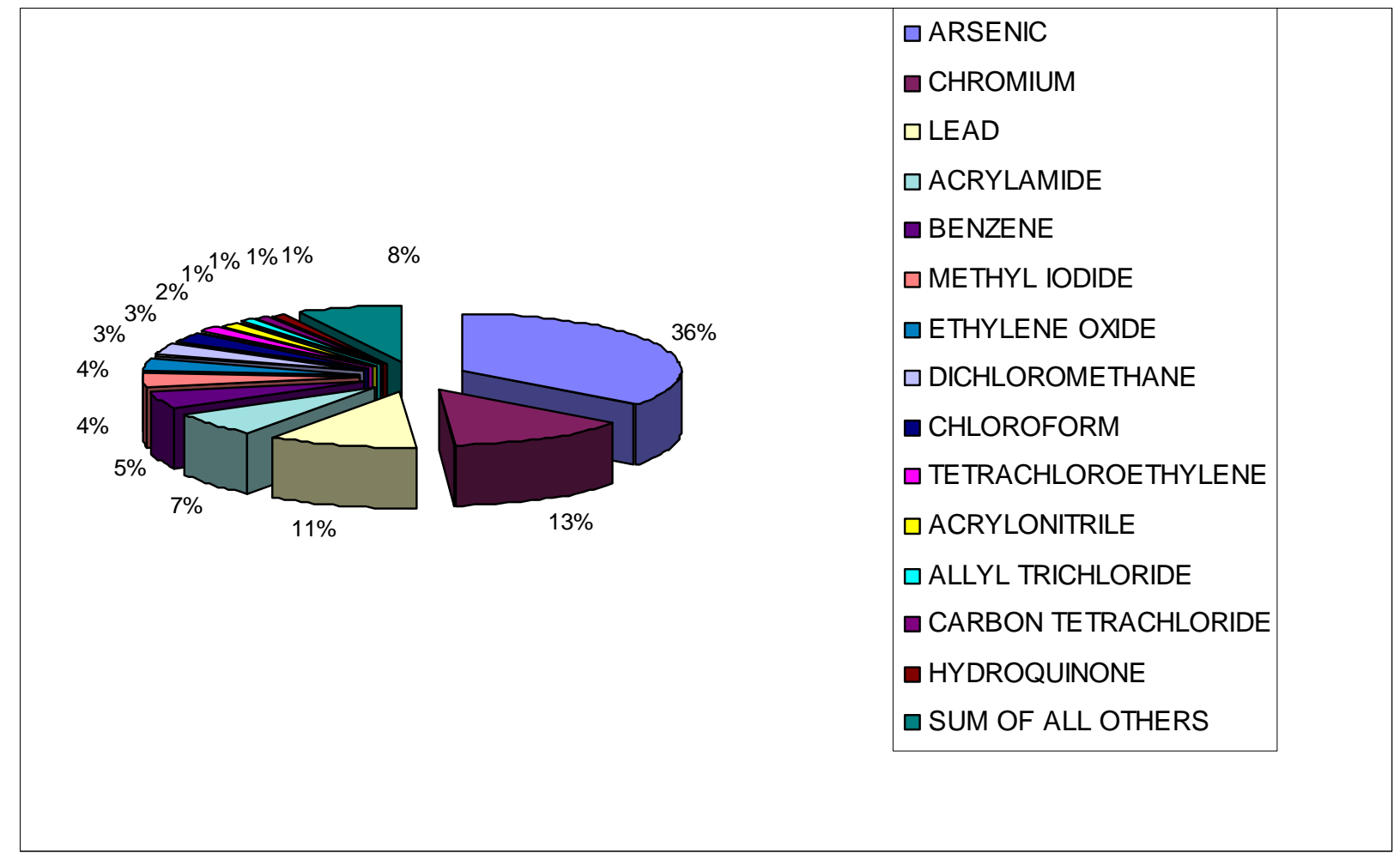

Figure 7. \% Contribution by Substance to the Air Normalized value for Human health cancer. Substances representing less than $1 \%$ were included within the "Sum of All Others."

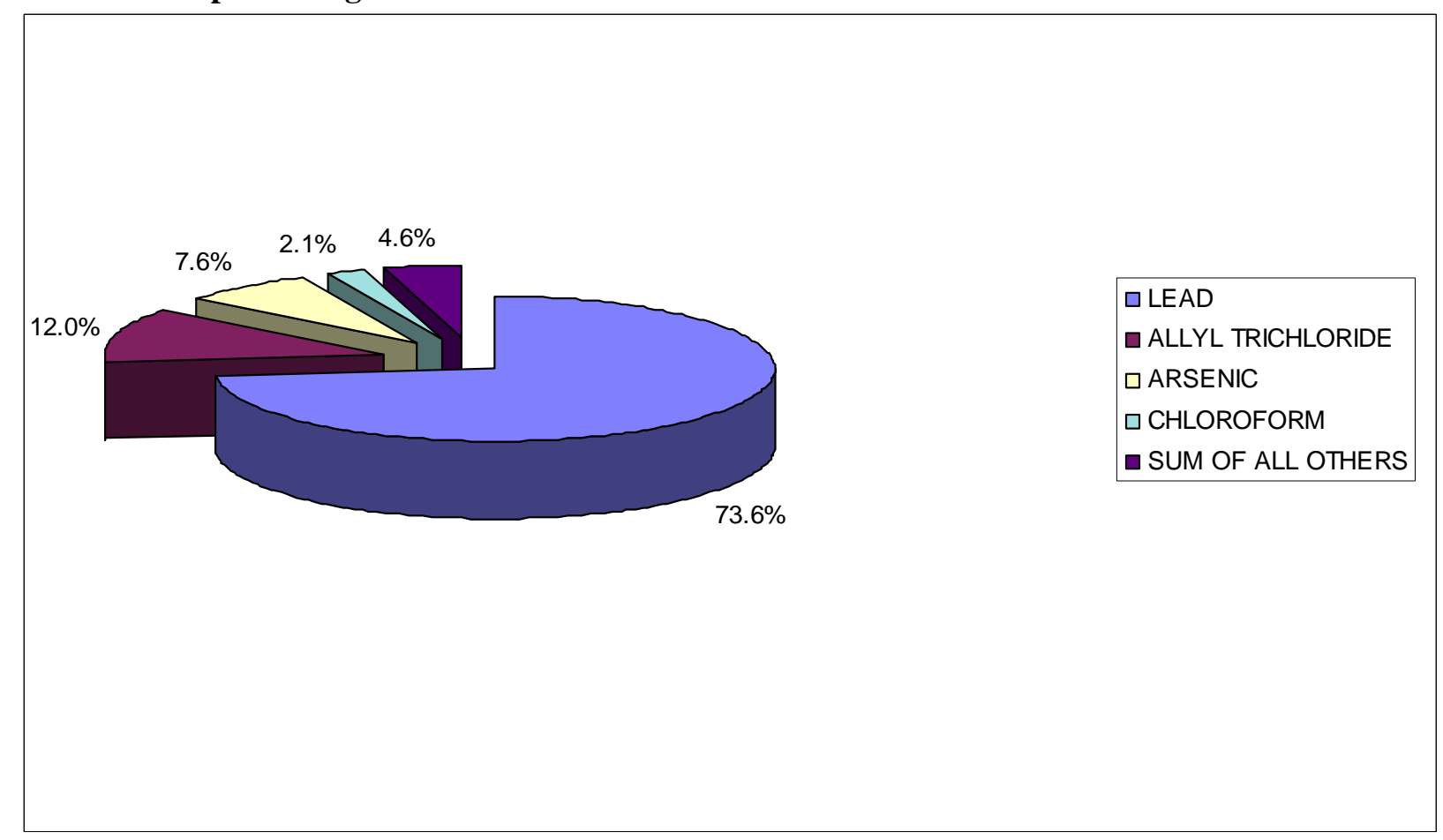

Figure 8. \% Contribution by Substance to the Water Normalized value for Human Health Cancer. Substances representing less than $1 \%$ were included within the "Sum of All Others." 


\begin{tabular}{|c|c|c|c|}
\hline Substance Name & $\begin{array}{l}\text { U.S. Normalized } \\
\text { Air Values } \\
\text { (Benzene eq/year) }\end{array}$ & $\begin{array}{c}\text { U.S. Normalized } \\
\text { Air Values Per } \\
\text { Capita (Benzene } \\
\text { eq/year per capita) }\end{array}$ & $\begin{array}{l}\text { Percentage } \\
\text { Contribution to } \\
\text { the Total Value }\end{array}$ \\
\hline ARSENIC & $2.50 \mathrm{E}+07$ & $8.97 \mathrm{E}-02$ & $35.61 \%$ \\
\hline CHROMIUM & $9.34 \mathrm{E}+06$ & $3.34 \mathrm{E}-02$ & $13.28 \%$ \\
\hline LEAD & $7.87 \mathrm{E}+06$ & $2.82 \mathrm{E}-02$ & $11.19 \%$ \\
\hline ACRYLAMIDE & $4.80 \mathrm{E}+06$ & $1.72 \mathrm{E}-02$ & $6.83 \%$ \\
\hline BENZENE & $3.58 \mathrm{E}+06$ & $1.28 \mathrm{E}-02$ & $5.09 \%$ \\
\hline METHYL IODIDE & $2.80 \mathrm{E}+06$ & $1.00 \mathrm{E}-02$ & $3.99 \%$ \\
\hline ETHYLENE OXIDE & $2.48 \mathrm{E}+06$ & $8.87 \mathrm{E}-03$ & $3.52 \%$ \\
\hline DICHLOROMETHANE & $2.31 \mathrm{E}+06$ & $8.26 \mathrm{E}-03$ & $3.28 \%$ \\
\hline CHLOROFORM & $1.94 \mathrm{E}+06$ & $6.96 \mathrm{E}-03$ & $2.76 \%$ \\
\hline TETRACHLOROETHYLENE & $1.32 \mathrm{E}+06$ & $4.72 \mathrm{E}-03$ & $1.87 \%$ \\
\hline ACRYLONITRILE & $9.45 \mathrm{E}+05$ & $3.39 \mathrm{E}-03$ & $1.34 \%$ \\
\hline ALLYL TRICHLORIDE & $8.30 \mathrm{E}+05$ & 2.97E-03 & $1.18 \%$ \\
\hline CARBON TETRACHLORIDE & $7.85 \mathrm{E}+05$ & $2.81 \mathrm{E}-03$ & $1.12 \%$ \\
\hline HYDROQUINONE & $7.73 \mathrm{E}+05$ & $2.77 \mathrm{E}-03$ & $1.10 \%$ \\
\hline DIMETHYL SULFATE & $6.51 \mathrm{E}+05$ & $2.33 \mathrm{E}-03$ & $0.93 \%$ \\
\hline VINYL CHLORIDE & $6.31 \mathrm{E}+05$ & $2.26 \mathrm{E}-03$ & $0.90 \%$ \\
\hline 1,2-DICHLOROETHANE & $5.71 \mathrm{E}+05$ & $2.04 \mathrm{E}-03$ & $0.81 \%$ \\
\hline 1,3-BUTADIENE & $3.88 \mathrm{E}+05$ & $1.39 \mathrm{E}-03$ & $0.55 \%$ \\
\hline METHYL CHLORIDE & $3.71 \mathrm{E}+05$ & $1.33 \mathrm{E}-03$ & $0.53 \%$ \\
\hline \begin{tabular}{|l|l|} 
ATRAZINE \\
\end{tabular} & $3.30 \mathrm{E}+05$ & $1.18 \mathrm{E}-03$ & $0.47 \%$ \\
\hline BENZIDINE & $3.19 \mathrm{E}+05$ & $1.14 \mathrm{E}-03$ & $0.45 \%$ \\
\hline TRICHLOROETHYLENE & $3.08 \mathrm{E}+05$ & $1.10 \mathrm{E}-03$ & $0.44 \%$ \\
\hline BENZOIC TRICHLORIDE & $1.93 \mathrm{E}+05$ & $6.93 \mathrm{E}-04$ & $0.28 \%$ \\
\hline NICKEL & $1.92 \mathrm{E}+05$ & $6.87 \mathrm{E}-04$ & $0.27 \%$ \\
\hline 4,4'-DIAMINO DITAN & $1.85 \mathrm{E}+05$ & $6.64 \mathrm{E}-04$ & $0.26 \%$ \\
\hline 1,1,2-TRICHLOROETHANE & $1.65 \mathrm{E}+05$ & $5.93 \mathrm{E}-04$ & $0.24 \%$ \\
\hline 1,2-DICHLOROPROPANE & $1.38 \mathrm{E}+05$ & 4.94E-04 & $0.20 \%$ \\
\hline BENZO(B)PYRIDINE & $1.11 \mathrm{E}+05$ & 3.97E-04 & $0.16 \%$ \\
\hline PROPYLENE OXIDE & $1.02 \mathrm{E}+05$ & $3.64 \mathrm{E}-04$ & $0.14 \%$ \\
\hline 1,4-DICHLOROBENZENE & $1.00 \mathrm{E}+05$ & $3.60 \mathrm{E}-04$ & $0.14 \%$ \\
\hline 1-CHLORO-2,3-EPOXYPROPANE & $8.51 \mathrm{E}+04$ & $3.05 \mathrm{E}-04$ & $0.12 \%$ \\
\hline SIMAZINE & $8.17 \mathrm{E}+04$ & $2.93 \mathrm{E}-04$ & $0.12 \%$ \\
\hline 2-NITROPROPANE & $6.96 \mathrm{E}+04$ & $2.49 \mathrm{E}-04$ & $0.10 \%$ \\
\hline CADMIUM & $4.87 \mathrm{E}+04$ & $1.74 \mathrm{E}-04$ & $0.07 \%$ \\
\hline HEXACHLOROETHANE & $3.68 \mathrm{E}+04$ & $1.32 \mathrm{E}-04$ & $0.05 \%$ \\
\hline NITROGLYCERIN & $3.66 \mathrm{E}+04$ & $1.31 \mathrm{E}-04$ & $0.05 \%$ \\
\hline 1,2-DIBROMOETHANE & $3.15 \mathrm{E}+04$ & $1.13 \mathrm{E}-04$ & $0.04 \%$ \\
\hline DICHLOROBENZENE (MIXED ISOMERS) & $3.12 \mathrm{E}+04$ & $1.12 \mathrm{E}-04$ & $0.04 \%$ \\
\hline HYDRAZINE & $3.07 \mathrm{E}+04$ & $1.10 \mathrm{E}-04$ & $0.04 \%$ \\
\hline DICOFOL & $2.79 \mathrm{E}+04$ & $9.98 \mathrm{E}-05$ & $0.04 \%$ \\
\hline 1,1-DICHLOROETHYLENE & $2.55 \mathrm{E}+04$ & $9.12 \mathrm{E}-05$ & $0.04 \%$ \\
\hline CYANAZINE & $2.48 \mathrm{E}+04$ & $8.87 \mathrm{E}-05$ & $0.04 \%$ \\
\hline ACETALDEHYDE & $2.39 \mathrm{E}+04$ & $8.58 \mathrm{E}-05$ & $0.03 \%$ \\
\hline HEXACHLOROBENZENE & $2.14 \mathrm{E}+04$ & $7.67 \mathrm{E}-05$ & $0.03 \%$ \\
\hline $1,1,2,2$-TETRACHLOROETHANE & $2.12 \mathrm{E}+04$ & $7.58 \mathrm{E}-05$ & $0.03 \%$ \\
\hline FORMALDEHYDE & $2.05 \mathrm{E}+04$ & $7.36 \mathrm{E}-05$ & $0.03 \%$ \\
\hline 2,4-DIAMINOTOLUENE & $1.48 \mathrm{E}+04$ & $5.31 \mathrm{E}-05$ & $0.02 \%$ \\
\hline BIS(2-ETHYLHEXYL)PHTHALATE & $1.03 \mathrm{E}+04$ & $3.69 \mathrm{E}-05$ & $0.01 \%$ \\
\hline BROMODICHLOROMETHANE & $9.65 \mathrm{E}+03$ & $3.46 \mathrm{E}-05$ & $0.01 \%$ \\
\hline 1,1-DICHLOROETHANE & $7.50 \mathrm{E}+03$ & $2.69 \mathrm{E}-05$ & $0.01 \%$ \\
\hline HEXACHLORO-1,3-BUTADIENE & $7.29 \mathrm{E}+03$ & $2.61 E-05$ & $0.01 \%$ \\
\hline BENZYL CHLORIDE & $6.66 \mathrm{E}+03$ & $2.39 \mathrm{E}-05$ & $0.01 \%$ \\
\hline LINURON & $6.35 \mathrm{E}+03$ & $2.28 \mathrm{E}-05$ & $0.01 \%$ \\
\hline PERMETHRIN & $6.00 \mathrm{E}+03$ & $2.15 \mathrm{E}-05$ & $0.01 \%$ \\
\hline 2,4-D [ACETIC ACID (2,4-DICHLOROPHENOXY)-] & $5.90 \mathrm{E}+03$ & $2.11 \mathrm{E}-05$ & $0.01 \%$ \\
\hline BIFENTHRIN & $5.51 \mathrm{E}+03$ & $1.97 \mathrm{E}-05$ & $0.01 \%$ \\
\hline TRIALLATE & $5.14 \mathrm{E}+03$ & $1.84 \mathrm{E}-05$ & $0.01 \%$ \\
\hline CHLOROMETHYL METHYL ETHER & $5.04 \mathrm{E}+03$ & $1.81 \mathrm{E}-05$ & $0.01 \%$ \\
\hline THIOUREA & $4.78 \mathrm{E}+03$ & $1.71 \mathrm{E}-05$ & $0.01 \%$ \\
\hline BERYLLIUM & $4.06 \mathrm{E}+03$ & $1.45 \mathrm{E}-05$ & $0.01 \%$ \\
\hline SUM OF ALL OTHERS & $2.82 \mathrm{E}+04$ & $1.01 \mathrm{E}-04$ & $0.04 \%$ \\
\hline TOTAL & $7.03 \mathrm{E}+07$ & $2.52 \mathrm{E}-01$ & $100.00 \%$ \\
\hline
\end{tabular}

Table 15. 1999 U.S. Normalized values by Substance for Human Health Cancer Air Estimates for all substance with $>0.01 \%$ contribution to total normalized value 


\begin{tabular}{|c|c|c|c|}
\hline Substance Name & $\begin{array}{l}\text { U.S. Normalized } \\
\text { Water Values } \\
\text { (Benzene eq/yr) }\end{array}$ & $\begin{array}{l}\text { U.S. Normalized } \\
\text { Water Values Per } \\
\text { Capita (Benzene } \\
\text { eq/yr per capita) }\end{array}$ & $\begin{array}{l}\text { Percentage } \\
\text { Contribution to } \\
\text { the Total Value }\end{array}$ \\
\hline LEAD & $1.30 \mathrm{E}+06$ & 4.64E-03 & $73.64 \%$ \\
\hline ALLYL TRICHLORIDE & $2.12 \mathrm{E}+05$ & 7.58E-04 & $12.03 \%$ \\
\hline ARSENIC & $1.34 \mathrm{E}+05$ & 4.82E-04 & $7.64 \%$ \\
\hline CHLOROFORM & $3.74 \mathrm{E}+04$ & 1.34E-04 & $2.12 \%$ \\
\hline 1,3-BUTADIENE & $1.33 E+04$ & 4.78E-05 & $0.76 \%$ \\
\hline BENZENE & $1.26 \mathrm{E}+04$ & 4.52E-05 & $0.72 \%$ \\
\hline 2-NITROPROPANE & $9.29 \mathrm{E}+03$ & 3.33E-05 & $0.53 \%$ \\
\hline 1,4-DIOXANE & $8.63 \mathrm{E}+03$ & 3.09E-05 & $0.49 \%$ \\
\hline 1,2-DICHLOROPROPANE & $8.12 \mathrm{E}+03$ & 2.91E-05 & $0.46 \%$ \\
\hline HYDRAZINE & $4.56 \mathrm{E}+03$ & 1.63E-05 & $0.26 \%$ \\
\hline HEXACHLOROBENZENE & $4.04 \mathrm{E}+03$ & 1.45E-05 & $0.23 \%$ \\
\hline ETHYLENE OXIDE & $3.35 \mathrm{E}+03$ & 1.20E-05 & $0.19 \%$ \\
\hline PROPYLENE OXIDE & $2.96 \mathrm{E}+03$ & 1.06E-05 & $0.17 \%$ \\
\hline CHLORDANE & $1.47 \mathrm{E}+03$ & 5.25E-06 & $0.08 \%$ \\
\hline 4,4'-DIAMINO DITAN & $1.32 \mathrm{E}+03$ & 4.73E-06 & $0.08 \%$ \\
\hline 1,2-DICHLOROETHANE & $1.24 \mathrm{E}+03$ & 4.44E-06 & $0.07 \%$ \\
\hline 1,1,2-TRICHLOROETHANE & $9.86 \mathrm{E}+02$ & 3.53E-06 & $0.06 \%$ \\
\hline ACRYLONITRILE & $9.54 \mathrm{E}+02$ & $3.42 E-06$ & $0.05 \%$ \\
\hline BIS(2-ETHYLHEXYL)PHTHALATE & $9.22 \mathrm{E}+02$ & 3.30E-06 & $0.05 \%$ \\
\hline ACETALDEHYDE & $8.79 \mathrm{E}+02$ & $3.15 E-06$ & $0.05 \%$ \\
\hline 1,4-DICHLOROBENZENE & $8.30 \mathrm{E}+02$ & 2.97E-06 & $0.05 \%$ \\
\hline DICHLOROMETHANE & $6.73 \mathrm{E}+02$ & 2.41E-06 & $0.04 \%$ \\
\hline TETRACHLOROETHYLENE & $6.38 \mathrm{E}+02$ & $2.28 \mathrm{E}-06$ & $0.04 \%$ \\
\hline CARBON TETRACHLORIDE & $3.48 \mathrm{E}+02$ & 1.25E-06 & $0.02 \%$ \\
\hline 1,3-DICHLOROBENZENE & $3.26 \mathrm{E}+02$ & 1.17E-06 & $0.02 \%$ \\
\hline BIS(2-CHLOROETHYL)ETHER & $3.16 \mathrm{E}+02$ & 1.13E-06 & $0.02 \%$ \\
\hline 1,1-DICHLOROETHYLENE & $2.95 \mathrm{E}+02$ & 1.06E-06 & $0.02 \%$ \\
\hline VINYL CHLORIDE & $2.76 \mathrm{E}+02$ & 9.89E-07 & $0.02 \%$ \\
\hline ACRYLAMIDE & $2.28 \mathrm{E}+02$ & 8.16E-07 & $0.01 \%$ \\
\hline METHYL CHLORIDE & $2.19 \mathrm{E}+02$ & $7.83 E-07$ & $0.01 \%$ \\
\hline METHYL IODIDE & $2.01 \mathrm{E}+02$ & 7.19E-07 & $0.01 \%$ \\
\hline BENZO(B)PYRIDINE & $1.98 \mathrm{E}+02$ & 7.08E-07 & $0.01 \%$ \\
\hline GAMMA-HCH (LINDANE) & $1.56 \mathrm{E}+02$ & 5.59E-07 & $0.01 \%$ \\
\hline SIMAZINE & $1.20 \mathrm{E}+02$ & $4.28 \mathrm{E}-07$ & $0.01 \%$ \\
\hline TRICHLOROETHYLENE & $1.07 \mathrm{E}+02$ & 3.82E-07 & $0.01 \%$ \\
\hline HEPTACHLOR & $1.04 \mathrm{E}+02$ & 3.73E-07 & $0.01 \%$ \\
\hline 1,2-DIBROMOETHANE & $9.96 \mathrm{E}+01$ & 3.57E-07 & $0.01 \%$ \\
\hline SUM OF ALL OTHERS & $4.63 \mathrm{E}+02$ & 1.66E-06 & $0.03 \%$ \\
\hline TOTAL & $1.76 \mathrm{E}+06$ & $6.30 \mathrm{E}-03$ & $100.00 \%$ \\
\hline
\end{tabular}

Table 16. 1999 U.S. Normalized values by Substance for Human Health Cancer Water Estimates

for all substance with $>0.01 \%$ contribution to total normalized value

HUMAN HEALTH NON-CANCER 


\begin{tabular}{|c|c|c|c|}
\hline Substance Name & \begin{tabular}{|c|} 
TRI Annual Air \\
Emissions Esimate \\
(kg/yr)
\end{tabular} & $\begin{array}{c}\text { NEI Annual } \\
\text { Emissions } \\
\text { Estimate (kg/yr) }\end{array}$ & Reference \\
\hline LEAD & $1.35 \mathrm{E}+05$ & $0.00 \mathrm{E}+00$ & $\mathrm{TRI}$ \\
\hline ANTIMONY & $4.93 \mathrm{E}+03$ & $0.00 \mathrm{E}+00$ & $\overline{T R I}$ \\
\hline COPPER & $7.04 \mathrm{E}+05$ & $0.00 \mathrm{E}+00$ & $\overline{T R I}$ \\
\hline NICKEL & $1.27 \mathrm{E}+05$ & $0.00 \mathrm{E}+00$ & $\overline{\mathrm{TRI}}$ \\
\hline ALUMINUM (FUME OR DUST) & $8.00 \mathrm{E}+05$ & $0.00 \mathrm{E}+00$ & $\mathrm{TRI}$ \\
\hline CHROMIUM & $1.34 \mathrm{E}+05$ & $0.00 \mathrm{E}+00$ & $\overline{T R I}$ \\
\hline ZINC & $6.39 \mathrm{E}+05$ & $0.00 \mathrm{E}+00$ & $\overline{\mathrm{TRI}}$ \\
\hline CARBON DISULFIDE & $1.63 \mathrm{E}+07$ & $1.44 \mathrm{E}+04$ & $\mathrm{TRI}$ \\
\hline THALLIUM & $1.43 E+03$ & $0.00 \mathrm{E}+00$ & $\overline{T R I}$ \\
\hline MANGANESE & $3.89 E+05$ & $0.00 \mathrm{E}+00$ & $\overline{\mathrm{TRI}}$ \\
\hline SELENIUM & $3.17 E+04$ & $0.00 \mathrm{E}+00$ & $\mathrm{TRI}$ \\
\hline ARSENIC & $2.95 \mathrm{E}+03$ & $0.00 \mathrm{E}+00$ & $\overline{T R I}$ \\
\hline BARIUM & $7.47 \mathrm{E}+04$ & $0.00 \mathrm{E}+00$ & TRI \\
\hline VANADIUM (FUME OR DUST) & $7.79 \mathrm{E}+03$ & $0.00 \mathrm{E}+00$ & $\overline{\mathrm{TRI}}$ \\
\hline HYDROCYANIC ACID & $7.33 \mathrm{E}+05$ & $0.00 \mathrm{E}+00$ & $\overline{T R I}$ \\
\hline MERCURY & $9.37 \mathrm{E}+03$ & $0.00 \mathrm{E}+00$ & $\overline{T R I}$ \\
\hline METHYL BROMIDE & $6.49 \mathrm{E}+05$ & $1.77 \mathrm{E}+04$ & $\overline{\mathrm{TRI}}$ \\
\hline CADMIUM & $1.95 \mathrm{E}+03$ & $0.00 \mathrm{E}+00$ & $\overline{T R I}$ \\
\hline COBALT & $1.15 \mathrm{E}+04$ & $0.00 \mathrm{E}+00$ & $\overline{T R I}$ \\
\hline AMMONIA & $7.01 \mathrm{E}+07$ & $0.00 \mathrm{E}+00$ & $\mathrm{TRI}$ \\
\hline ACROLEIN & $9.29 \mathrm{E}+04$ & $1.33 \mathrm{E}+04$ & $\overline{T R I}$ \\
\hline SILVER & $5.53 \mathrm{E}+03$ & $0.00 \mathrm{E}+00$ & $\overline{T R I}$ \\
\hline CARBON TETRACHLORIDE & $1.06 \mathrm{E}+05$ & $8.78 \mathrm{E}+02$ & $\mathrm{TRI}$ \\
\hline ETHYLENE OXIDE & $2.25 \mathrm{E}+05$ & $5.60 \mathrm{E}+02$ & $\overline{T R I}$ \\
\hline TETRACHLOROETHYLENE & $1.83 \mathrm{E}+06$ & $2.12 \mathrm{E}+04$ & $\overline{T R I}$ \\
\hline ACRYLAMIDE & $1.13 \mathrm{E}+04$ & $8.04 \mathrm{E}+00$ & $\mathrm{TRI}$ \\
\hline CARBONYL CHLORIDE & $7.56 \mathrm{E}+03$ & $2.77 \mathrm{E}+00$ & $\overline{T R I}$ \\
\hline 1-CHLORO-2,3-EPOXYPROPANE & $7.07 \mathrm{E}+04$ & $5.19 \mathrm{E}+01$ & $\overline{T R I}$ \\
\hline MALEIC ANHYDRIDE & $1.72 \mathrm{E}+05$ & $4.63 \mathrm{E}+02$ & $\overline{\mathrm{TRI}}$ \\
\hline HYDROCHLORIC ACID & $3.04 \mathrm{E}+08$ & $2.27 \mathrm{E}+05$ & TRI \\
\hline BERYLLIUM & $3.49 \mathrm{E}+02$ & $0.00 \mathrm{E}+00$ & $\overline{T R I}$ \\
\hline 1,2-DICHLOROPROPANE & $1.13 \mathrm{E}+05$ & $7.10 \mathrm{E}+01$ & $\overline{T R I}$ \\
\hline BENZENE & $3.58 \mathrm{E}+06$ & $1.59 \mathrm{E}+05$ & $\mathrm{TRI}$ \\
\hline METHYL CHLORIDE & $1.27 \mathrm{E}+06$ & $1.61 \mathrm{E}+04$ & $\overline{T R I}$ \\
\hline TOLUENE & $4.15 \mathrm{E}+07$ & $4.52 \mathrm{E}+05$ & $\mathrm{TRI}$ \\
\hline ACRYLONITRILE & $4.47 E+05$ & $5.88 \mathrm{E}+02$ & $\overline{T R I}$ \\
\hline VINYL CHLORIDE & $3.88 \mathrm{E}+05$ & $6.07 \mathrm{E}+02$ & $\overline{T R I}$ \\
\hline CHLOROFORM & $2.39 E+06$ & $6.87 E+03$ & $\mathrm{TRI}$ \\
\hline FORMALDEHYDE & $5.63 E+06$ & $1.34 \mathrm{E}+05$ & $\overline{\mathrm{TRI}}$ \\
\hline ACETALDEHYDE & $5.48 \mathrm{E}+06$ & $4.21 \mathrm{E}+04$ & $\overline{T R I}$ \\
\hline DIETHANOLAMINE & $1.71 \mathrm{E}+05$ & $1.29 \mathrm{E}+02$ & $\overline{T R I}$ \\
\hline ACETONITRILE & $4.02 \mathrm{E}+05$ & $1.22 \mathrm{E}+03$ & $\overline{T R I}$ \\
\hline DIAZINON & $1.20 \mathrm{E}+04$ & $0.00 \mathrm{E}+00$ & $\overline{T R I}$ \\
\hline
\end{tabular}

Table 17. 1999 U.S. Annual Air Emissions Estimates for Human Health Noncancer Substances whose Normalized Contribution Represent $>\mathbf{0 . 0 1 \%}$ of the Total Normalized Value 


\begin{tabular}{|l|c|c|}
\hline \multicolumn{1}{|c|}{ Substance Name } & $\begin{array}{c}\text { TRI Annual Water } \\
\text { Emissions Esimate } \\
(\mathbf{k g} / \mathbf{y r})\end{array}$ & $\begin{array}{c}\text { Reference } \\
\text { Number }\end{array}$ \\
\hline LEAD & $3.70 \mathrm{E}+03$ & TRI \\
\hline CADMIUM & $1.32 \mathrm{E}+02$ & TRI \\
\hline COPPER & $1.66 \mathrm{E}+04$ & TRI \\
\hline MERCURY & $6.03 \mathrm{E}+01$ & TRI \\
\hline ANTIMONY & $6.24 \mathrm{E}+03$ & TRI \\
\hline THALLIUM & $2.72 \mathrm{E}+02$ & TRI \\
\hline CHROMIUM & $1.45 \mathrm{E}+04$ & TRI \\
\hline ARSENIC & $4.77 \mathrm{E}+02$ & TRI \\
\hline S,S,S-TRIBUTYLTRITHIOPHOSPHATE & $7.30 \mathrm{E}+01$ & TRI \\
\hline 1,2-DICHLOROPROPANE & $4.19 \mathrm{E}+03$ & TRI \\
\hline
\end{tabular}

Table 18. 1999 U.S. Annual Water Emissions Estimates for Human Health Noncancer Substances whose Normalized Contribution Represent $\mathbf{> 0 . 0 1 \%}$ of the Total Normalized Value

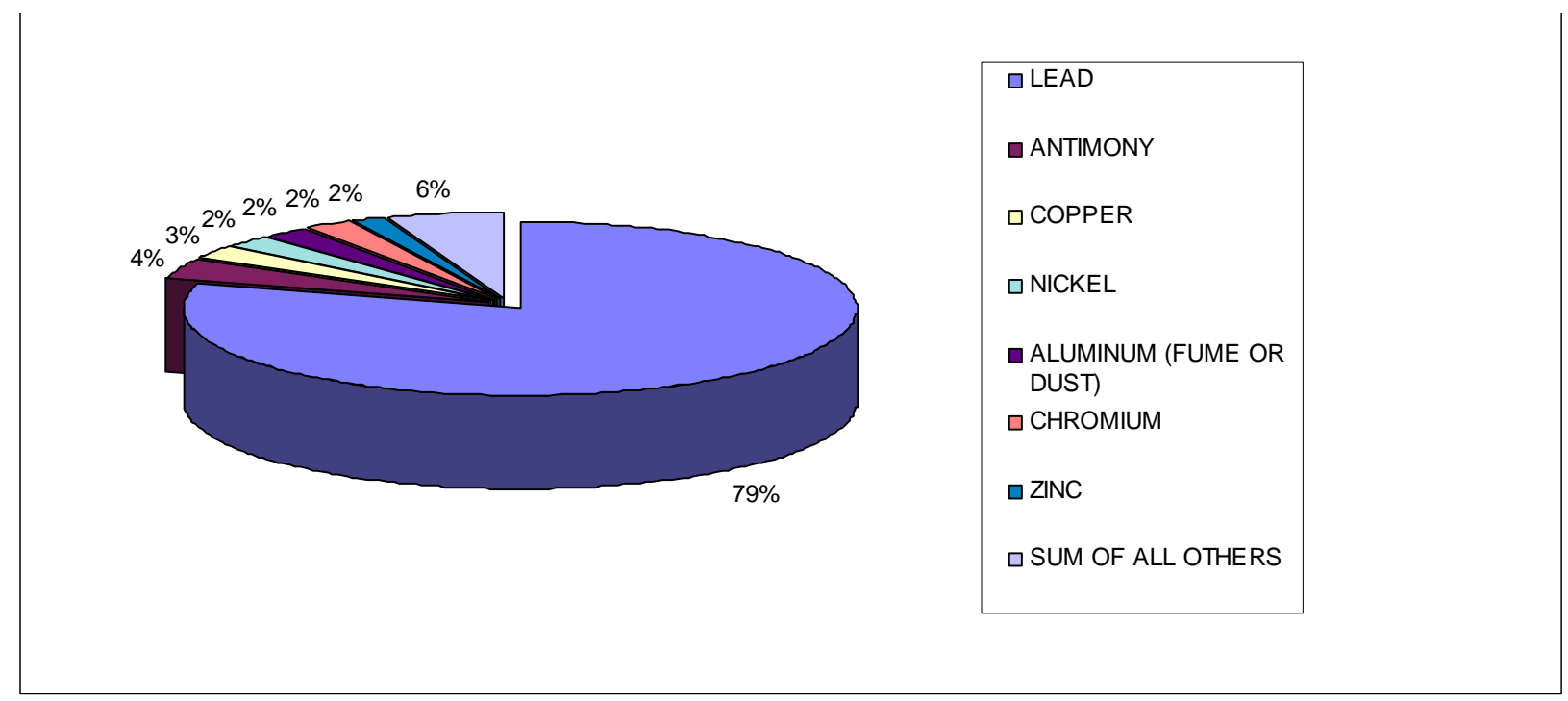

Figure 9. \% Contribution by Substance to the Air Normalized value for Human health Noncancer. Substances representing less than $1 \%$ were included within the "Sum of All Others." 


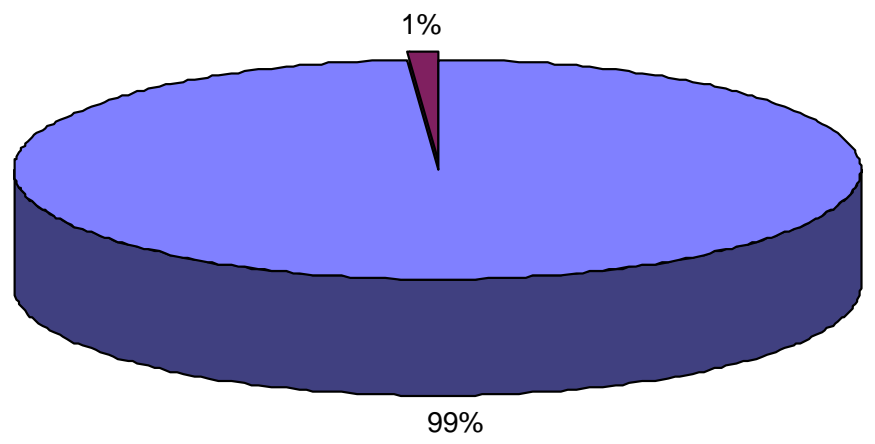

$\square$ LEAD

$\square$ SUM OF ALL OTHERS

Figure 10. \% Contribution by Substance to the Water Normalized value for Human health Noncancer. Substances representing less than $1 \%$ were included within the "Sum of All Others." 


\begin{tabular}{|c|c|c|c|}
\hline Substance Name & $\begin{array}{l}\text { U.S. Normalized } \\
\text { Air Values } \\
\text { (Toluene eq/yr) }\end{array}$ & $\begin{array}{l}\text { U.S. Normalized } \\
\text { Air Value Per } \\
\text { Capita (Toluene } \\
\text { eq/yr per capita) }\end{array}$ & $\begin{array}{l}\text { Percentage } \\
\text { Contribution to } \\
\text { the Total Value }\end{array}$ \\
\hline LEAD & $2.94 \mathrm{E}+11$ & $1.05 \mathrm{E}+03$ & $79.48 \%$ \\
\hline ANTIMONY & $1.38 \mathrm{E}+10$ & $4.95 \mathrm{E}+01$ & $3.74 \%$ \\
\hline COPPER & $9.31 \mathrm{E}+09$ & $3.33 \mathrm{E}+01$ & $2.52 \%$ \\
\hline NICKEL & $9.12 \mathrm{E}+09$ & $3.27 \mathrm{E}+01$ & $2.47 \%$ \\
\hline ALUMINUM (FUME OR DUST) & $8.94 \mathrm{E}+09$ & $3.20 \mathrm{E}+01$ & $2.42 \%$ \\
\hline CHROMIUM & 7.70E+09 & $2.76 \mathrm{E}+01$ & $2.09 \%$ \\
\hline ZINC & $6.55 \mathrm{E}+09$ & $2.35 \mathrm{E}+01$ & $1.77 \%$ \\
\hline CARBON DISULFIDE & $3.07 \mathrm{E}+09$ & $1.10 \mathrm{E}+01$ & $0.83 \%$ \\
\hline THALLIUM & $2.75 \mathrm{E}+09$ & $9.85 \mathrm{E}+00$ & $0.74 \%$ \\
\hline MANGANESE & $2.37 \mathrm{E}+09$ & $8.50 \mathrm{E}+00$ & $0.64 \%$ \\
\hline SELENIUM & $2.26 \mathrm{E}+09$ & $8.09 \mathrm{E}+00$ & $0.61 \%$ \\
\hline ARSENIC & $1.38 \mathrm{E}+09$ & $4.95 \mathrm{E}+00$ & $0.37 \%$ \\
\hline BARIUM & $1.35 \mathrm{E}+09$ & $4.83 \mathrm{E}+00$ & $0.37 \%$ \\
\hline VANADIUM (FUME OR DUST) & $1.07 \mathrm{E}+09$ & $3.82 \mathrm{E}+00$ & $0.29 \%$ \\
\hline HYDROCYANIC ACID & $1.01 \mathrm{E}+09$ & $3.61 \mathrm{E}+00$ & $0.27 \%$ \\
\hline MERCURY & $9.36 \mathrm{E}+08$ & $3.35 \mathrm{E}+00$ & $0.25 \%$ \\
\hline METHYL BROMIDE & $7.59 \mathrm{E}+08$ & $2.72 \mathrm{E}+00$ & $0.21 \%$ \\
\hline CADMIUM & $7.55 \mathrm{E}+08$ & $2.70 \mathrm{E}+00$ & $0.20 \%$ \\
\hline COBALT & $3.35 \mathrm{E}+08$ & $1.20 \mathrm{E}+00$ & $0.09 \%$ \\
\hline AMMONIA & $2.25 \mathrm{E}+08$ & 8.05E-01 & $0.06 \%$ \\
\hline ACROLEIN & $2.20 \mathrm{E}+08$ & 7.87E-01 & $0.06 \%$ \\
\hline SILVER & $2.18 \mathrm{E}+08$ & $7.79 \mathrm{E}-01$ & $0.06 \%$ \\
\hline CARBON TETRACHLORIDE & 1.87E+08 & 6.69E-01 & $0.05 \%$ \\
\hline ETHYLENE OXIDE & $1.39 \mathrm{E}+08$ & 4.98E-01 & $0.04 \%$ \\
\hline TETRACHLOROETHYLENE & $1.37 \mathrm{E}+08$ & 4.92E-01 & $0.04 \%$ \\
\hline ACRYLAMIDE & $1.33 \mathrm{E}+08$ & 4.75E-01 & $0.04 \%$ \\
\hline CARBONYL CHLORIDE & $1.08 \mathrm{E}+08$ & 3.85E-01 & $0.03 \%$ \\
\hline 1-CHLORO-2,3-EPOXYPROPANE & $9.14 \mathrm{E}+07$ & 3.27E-01 & $0.02 \%$ \\
\hline MALEIC ANHYDRIDE & $6.94 \mathrm{E}+07$ & 2.49E-01 & $0.02 \%$ \\
\hline HYDROCHLORIC ACID & $6.39 \mathrm{E}+07$ & $2.29 \mathrm{E}-01$ & $0.02 \%$ \\
\hline BERYLLIUM & $5.84 \mathrm{E}+07$ & 2.09E-01 & $0.02 \%$ \\
\hline 1,2-DICHLOROPROPANE & $5.46 \mathrm{E}+07$ & 1.95E-01 & $0.01 \%$ \\
\hline BENZENE & $5.23 \mathrm{E}+07$ & 1.87E-01 & $0.01 \%$ \\
\hline METHYL CHLORIDE & $4.99 \mathrm{E}+07$ & $1.79 \mathrm{E}-01$ & $0.01 \%$ \\
\hline TOLUENE & $4.15 \mathrm{E}+07$ & $1.49 \mathrm{E}-01$ & $0.01 \%$ \\
\hline ACRYLONITRILE & $4.12 \mathrm{E}+07$ & $1.47 \mathrm{E}-01$ & $0.01 \%$ \\
\hline VINYL CHLORIDE & 4.08E+07 & 1.46E-01 & $0.01 \%$ \\
\hline CHLOROFORM & $2.98 \mathrm{E}+07$ & $1.07 \mathrm{E}-01$ & $0.01 \%$ \\
\hline FORMALDEHYDE & $2.85 \mathrm{E}+07$ & $1.02 \mathrm{E}-01$ & $0.01 \%$ \\
\hline ACETALDEHYDE & $2.33 \mathrm{E}+07$ & 8.34E-02 & $0.01 \%$ \\
\hline DIETHANOLAMINE & $2.17 \mathrm{E}+07$ & 7.78E-02 & $0.01 \%$ \\
\hline ACETONITRILE & 2.03E+07 & 7.27E-02 & $0.01 \%$ \\
\hline DIAZINON & $1.85 \mathrm{E}+07$ & $6.64 \mathrm{E}-02$ & $0.01 \%$ \\
\hline SUM OF ALL OTHERS & $2.67 \mathrm{E}+08$ & 9.55E-01 & $0.07 \%$ \\
\hline TOTAL & $3.69 E+11$ & $1.32 E+03$ & $100.00 \%$ \\
\hline
\end{tabular}

Table 19. 1999 U.S. Normalized values by Substance for Human Health Noncancer Air Estimates for all substance with $>\mathbf{0 . 0 1 \%}$ contribution to total normalized value. 


\begin{tabular}{|l|c|c|c|}
\hline \multicolumn{1}{|c|}{ Substance Name } & $\begin{array}{c}\text { U.S. Normalized } \\
\text { Water Values } \\
\text { (Toluene eq/yr) }\end{array}$ & $\begin{array}{c}\text { U.S. Normalized } \\
\text { Water Values Per } \\
\text { Capita (Toluene } \\
\text { eq/year per capita) }\end{array}$ & $\begin{array}{c}\text { Percentage } \\
\text { Contribution to } \\
\text { the Total Value }\end{array}$ \\
\hline LEAD & $4.19 \mathrm{E}+10$ & $1.50 \mathrm{E}+02$ & $98.82 \%$ \\
\hline CADMIUM & $2.66 \mathrm{E}+08$ & $9.52 \mathrm{E}-01$ & $0.63 \%$ \\
\hline COPPER & $9.78 \mathrm{E}+07$ & $3.50 \mathrm{E}-01$ & $0.23 \%$ \\
\hline MERCURY & $5.69 \mathrm{E}+07$ & $2.04 \mathrm{E}-01$ & $0.13 \%$ \\
\hline ANTIMONY & $2.62 \mathrm{E}+07$ & $9.40 \mathrm{E}-02$ & $0.06 \%$ \\
\hline THALLIUM & $1.74 \mathrm{E}+07$ & $6.24 \mathrm{E}-02$ & $0.04 \%$ \\
\hline CHROMIUM & $8.43 \mathrm{E}+06$ & $3.02 \mathrm{E}-02$ & $0.02 \%$ \\
\hline ARSENIC & $6.44 \mathrm{E}+06$ & $2.31 \mathrm{E}-02$ & $0.02 \%$ \\
\hline S,S,S-TRIBUTYLTRITHIOPHOSPHATE & $4.77 \mathrm{E}+06$ & $1.71 \mathrm{E}-02$ & $0.01 \%$ \\
\hline $1,2-\mathrm{DICHLOROPROPANE}$ & $2.31 \mathrm{E}+06$ & $8.28 \mathrm{E}-03$ & $0.01 \%$ \\
\hline SUM OF ALL OTHERS & $1.38 \mathrm{E}+07$ & $4.95 \mathrm{E}-02$ & $0.03 \%$ \\
\hline TOTAL & $4.24 \mathrm{E}+10$ & $1.52 \mathrm{E}+02$ & $100.00 \%$ \\
\hline
\end{tabular}

Table 20. 1999 U.S. Normalized values by Substance for Human Health Noncancer Water

Estimates for all substance with $>\mathbf{0 . 0 1 \%}$ contribution to total normalized value.

HUMAN HEALTH CRITERIA

\begin{tabular}{|l|c|c|}
\hline \multicolumn{1}{|c|}{ Substance } & $\begin{array}{c}\text { Total } \\
\text { Emissions } \\
\text { (kg/year) }\end{array}$ & Reference \\
\hline PM 10 & $2.13 \mathrm{E}+10$ & NEI \\
\hline PM 2.5 & $6.65 \mathrm{E}+09$ & NEI \\
\hline Sulfur Dioxide & $1.59 \mathrm{E}+10$ & NEI \\
\hline Nitrogen Oxides & $1.69 \mathrm{E}+10$ & NEI \\
\hline
\end{tabular}

Table 21. Human Health Criteria Substances with U.S. Annual Emissions Estimates

Results

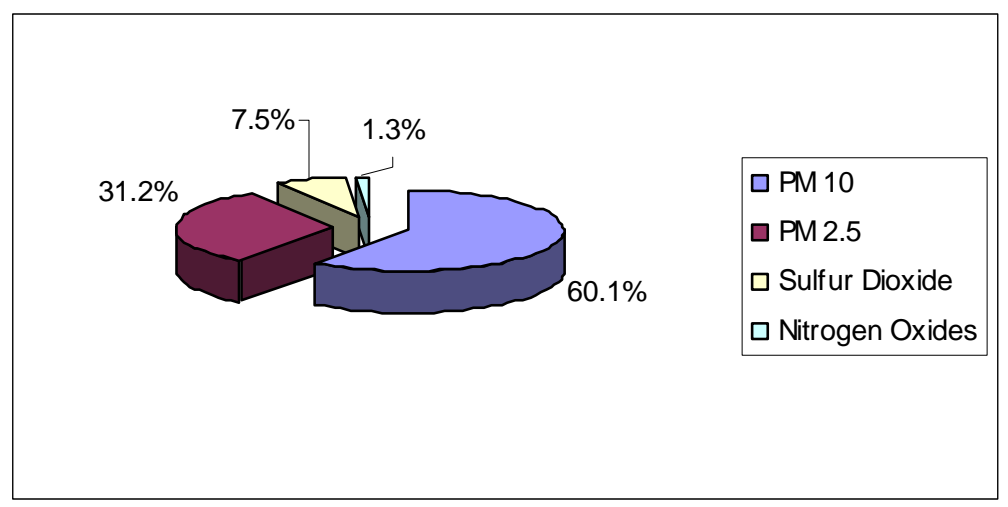

Figure 11. Percent Contribution by Substance to the Normalized value for Human Health Criteria 


\begin{tabular}{|l|c|r|r|}
\hline \multicolumn{1}{|c|}{ Substance } & $\begin{array}{c}\text { U.S. } \\
\text { Normalized } \\
\text { Values (PM } \\
\mathbf{2 . 5} \text { eq/yr) }\end{array}$ & $\begin{array}{c}\text { U.S. Normalized } \\
\text { Values (PM2.5 } \\
\text { eq/yr per capita) }\end{array}$ & $\begin{array}{c}\text { Percent } \\
\text { Contribution } \\
\text { to Total }\end{array}$ \\
\hline PM 10 & $1.28 \mathrm{E}+10$ & $4.58 \mathrm{E}+01$ & $60.1 \%$ \\
\hline PM 2.5 & $6.65 \mathrm{E}+09$ & $2.38 \mathrm{E}+01$ & $31.2 \%$ \\
\hline Sulfur Dioxide & $1.59 \mathrm{E}+09$ & $5.70 \mathrm{E}+00$ & $7.5 \%$ \\
\hline Nitrogen Oxides & $2.69 \mathrm{E}+08$ & $9.64 \mathrm{E}-01$ & $1.3 \%$ \\
\hline Total & $2.13 \mathrm{E}+10$ & $7.63 \mathrm{E}+01$ & $100.0 \%$ \\
\hline
\end{tabular}

Table 22. 1999 U.S. Normalized values by Substance for Human Health Criteria

\section{OZONE DEPLETION}

\begin{tabular}{|c|c|c|c|c|}
\hline Chemical Name & $\begin{array}{l}\text { TRI Annual Air } \\
\text { Emissions } \\
\text { Estimate (kg/yr) }\end{array}$ & $\begin{array}{l}\text { GHG Inventory } \\
\text { Emissions (kg/yr) }\end{array}$ & $\begin{array}{l}\text { NEI Emissions } \\
(\mathbf{k g} / \mathbf{y r})\end{array}$ & Data Source \\
\hline 1,1,1-Trichloroethane & $1.86 \mathrm{E}+05$ & $0.00 \mathrm{E}+00$ & $3.72 \mathrm{E}+04$ & TRI \\
\hline Carbon Tetrachloride & $1.06 \mathrm{E}+05$ & $2.35 \mathrm{E}+07$ & $8.78 \mathrm{E}+02$ & GHG Inv \\
\hline CFC 11 (TRICHLOROFLUOROMETHANE) & $1.83 \mathrm{E}+05$ & $6.60 \mathrm{E}+06$ & $0.00 \mathrm{E}+00$ & GHG Inv \\
\hline CFC 113 (FREON 113) & $3.82 \mathrm{E}+05$ & $0.00 \mathrm{E}+00$ & $0.00 \mathrm{E}+00$ & TRI \\
\hline CFC 114 & $4.24 \mathrm{E}+05$ & $0.00 \mathrm{E}+00$ & $0.00 \mathrm{E}+00$ & TRI \\
\hline CFC 115 & $3.48 \mathrm{E}+04$ & $1.60 \mathrm{E}+06$ & $0.00 \mathrm{E}+00$ & GHG Inv \\
\hline CFC 12 (DICHLOROFLUOROMETHANE) & $3.34 \mathrm{E}+05$ & $5.07 \mathrm{E}+07$ & $0.00 \mathrm{E}+00$ & GHG Inv \\
\hline CFC 13 (CHLOROTRIFLUOROMETHANE) & $4.89 \mathrm{E}+03$ & $0.00 \mathrm{E}+00$ & $0.00 \mathrm{E}+00$ & TRI \\
\hline $\begin{array}{l}\text { Halon } 1211 \\
\text { (BROMOCHLORODIFLUOROMETHANE) }\end{array}$ & $1.80 \mathrm{E}+03$ & $0.00 \mathrm{E}+00$ & $0.00 \mathrm{E}+00$ & TRI \\
\hline $\begin{array}{l}\text { Halon } 1301 \\
\text { (BROMOTRIFLUOROMETHANE) }\end{array}$ & $1.40 \mathrm{E}+04$ & $5.00 \mathrm{E}+05$ & $0.00 \mathrm{E}+00$ & GHG Inv \\
\hline HCFC 123 & $4.78 \mathrm{E}+04$ & $2.00 \mathrm{E}+06$ & $0.00 \mathrm{E}+00$ & GHG Inv \\
\hline HCFC 124 & $2.74 \mathrm{E}+05$ & $4.30 \mathrm{E}+06$ & $0.00 \mathrm{E}+00$ & GHG Inv \\
\hline HCFC 141B & $3.68 \mathrm{E}+06$ & $7.60 \mathrm{E}+06$ & $0.00 \mathrm{E}+00$ & GHG Inv \\
\hline HCFC 142B & $2.56 \mathrm{E}+06$ & $4.40 \mathrm{E}+06$ & $0.00 \mathrm{E}+00$ & GHG Inv \\
\hline HCFC 22 & $3.95 \mathrm{E}+06$ & $6.59 \mathrm{E}+07$ & $0.00 \mathrm{E}+00$ & GHG Inv \\
\hline HCFC 225CA & $1.63 \mathrm{E}+04$ & $0.00 \mathrm{E}+00$ & $0.00 \mathrm{E}+00$ & TRI \\
\hline HCFC 225CB & $1.96 \mathrm{E}+04$ & $0.00 \mathrm{E}+00$ & $0.00 \mathrm{E}+00$ & TRI \\
\hline Methyl Bromide & $6.49 \mathrm{E}+05$ & $0.00 \mathrm{E}+00$ & $1.77 \mathrm{E}+04$ & TRI \\
\hline
\end{tabular}

Table 23. Ozone Depleting Substances with U.S. Annual Emissions Estimates 


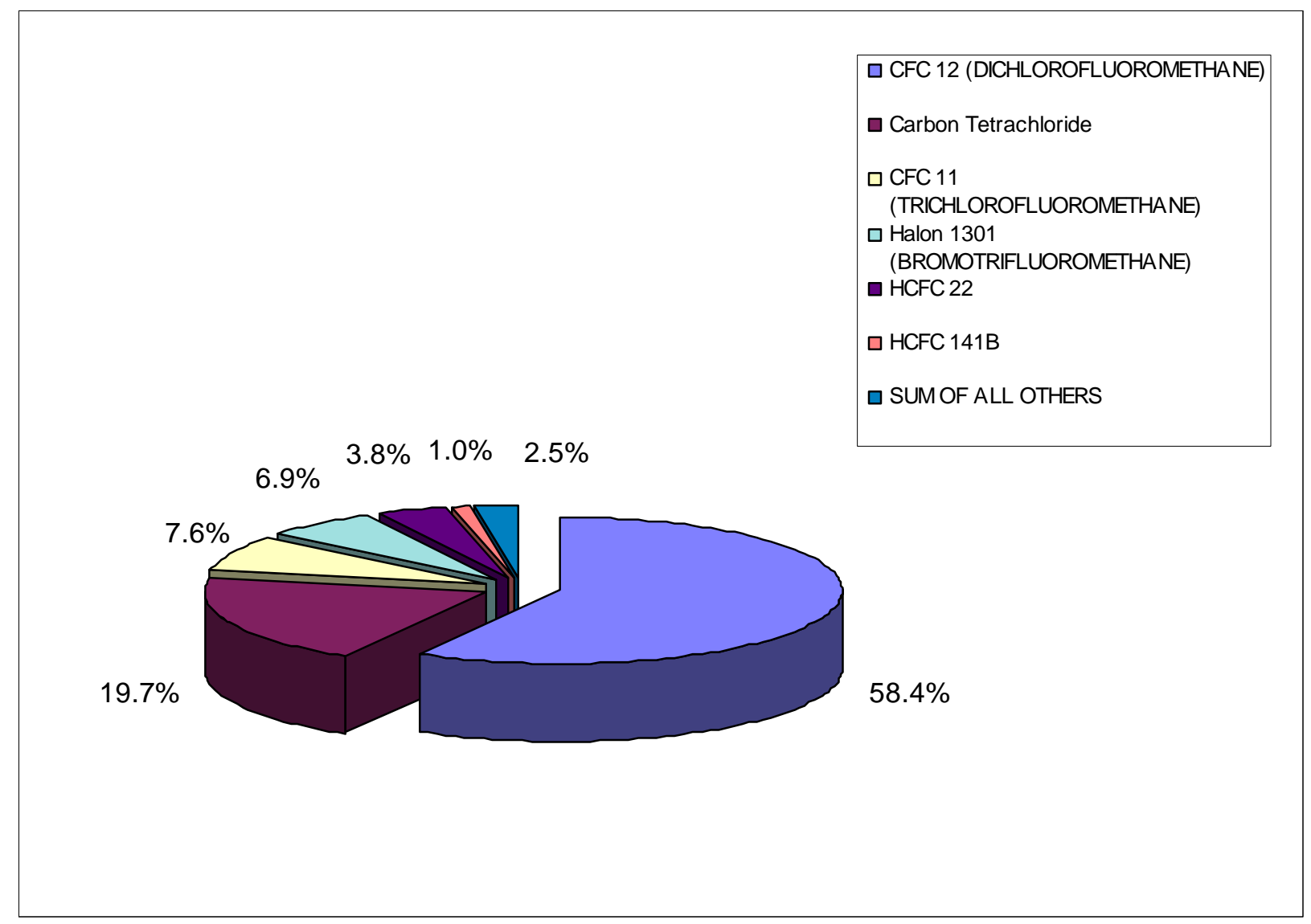

Figure 12. Percent Contribution by Substance to the Normalized value for Ozone Depleting Substances. 


\begin{tabular}{|l|c|c|c|}
\hline \multicolumn{1}{|c|}{ Chemical Name } & $\begin{array}{c}\text { U.S. Normalized } \\
\text { Value (CFC-11 } \\
\text { eq/yr) }\end{array}$ & $\begin{array}{c}\text { U.S. Normalized } \\
\text { Value Per Capita } \\
\text { (CFC-11 eq/yr per } \\
\text { capita) }\end{array}$ & $\begin{array}{c}\text { Percentage } \\
\text { Contribution to the } \\
\text { Total Value }\end{array}$ \\
\hline CFC 12 (DICHLOROFLUOROMETHANE) & $5.07 \mathrm{E}+07$ & $1.82 \mathrm{E}-01$ & $58.37 \%$ \\
\hline Carbon Tetrachloride & $1.72 \mathrm{E}+07$ & $6.15 \mathrm{E}-02$ & $19.75 \%$ \\
\hline CFC 11 (TRICHLOROFLUOROMETHANE) & $6.60 \mathrm{E}+06$ & $2.36 \mathrm{E}-02$ & $7.60 \%$ \\
\hline $\begin{array}{l}\text { Halon 1301 } \\
\text { (BROMOTRIFLUOROMETHANE) }\end{array}$ & $6.00 \mathrm{E}+06$ & $2.15 \mathrm{E}-02$ & $6.91 \%$ \\
\hline HCFC 22 & $3.30 \mathrm{E}+06$ & $1.18 \mathrm{E}-02$ & $3.79 \%$ \\
\hline HCFC 141B & $9.12 \mathrm{E}+05$ & $3.27 \mathrm{E}-03$ & $1.05 \%$ \\
\hline CFC 115 & $7.04 \mathrm{E}+05$ & $2.52 \mathrm{E}-03$ & $0.81 \%$ \\
\hline CFC 114 & $3.98 \mathrm{E}+05$ & $1.43 \mathrm{E}-03$ & $0.46 \%$ \\
\hline CFC 113 (FREON 113) & $3.82 \mathrm{E}+05$ & $1.37 \mathrm{E}-03$ & $0.44 \%$ \\
\hline HCFC 142B & $3.08 \mathrm{E}+05$ & $1.10 \mathrm{E}-03$ & $0.35 \%$ \\
\hline Methyl Bromide & $2.47 \mathrm{E}+05$ & $8.84 \mathrm{E}-04$ & $0.28 \%$ \\
\hline HCFC 124 & $8.60 \mathrm{E}+04$ & $3.08 \mathrm{E}-04$ & $0.10 \%$ \\
\hline HCFC 123 & $4.00 \mathrm{E}+04$ & $1.43 \mathrm{E}-04$ & $0.05 \%$ \\
\hline $1,1,1-T r i c h l o r o e t h a n e$ & $2.24 \mathrm{E}+04$ & $8.01 \mathrm{E}-05$ & $0.03 \%$ \\
\hline Halon 1211 & & & \\
\hline BROMOCHLORODIFLUOROMETHANE) & $1.08 \mathrm{E}+04$ & $3.88 \mathrm{E}-05$ & $0.01 \%$ \\
\hline CFC 13 (CHLOROTRIFLUOROMETHANE) & $4.89 \mathrm{E}+03$ & $1.75 \mathrm{E}-05$ & $0.01 \%$ \\
\hline HCFC 225CB & $5.89 \mathrm{E}+02$ & $2.11 \mathrm{E}-06$ & $0.00 \%$ \\
\hline HCFC 225CA & $3.27 \mathrm{E}+02$ & $1.17 \mathrm{E}-06$ & $0.00 \%$ \\
\hline TOTAL & $8.69 \mathrm{E}+07$ & $3.11 \mathrm{E}-01$ & $100.00 \%$ \\
\hline
\end{tabular}

Table 24. 1999 U.S. Normalized values by Substance for Ozone Depletion Potential

PHOTOCHEMICAL SMOG FORMATION

\begin{tabular}{|l|c|c|}
\hline \multicolumn{1}{|c|}{ Substance Name } & $\begin{array}{c}\text { U.S. Annual } \\
\text { Emissions Estimate } \\
\text { (kg/yr) }\end{array}$ & Reference \\
\hline Nitrogen Oxides (NOx) & $2.06 \mathrm{E}+10$ & $\mathrm{NEI}$ \\
\hline VOCs & $1.69 \mathrm{E}+10$ & $\mathrm{NEI}$ \\
\hline
\end{tabular}

Table 25. Photochemical Smog Formation Pollutants with U.S. Annual Emissions Estimates 


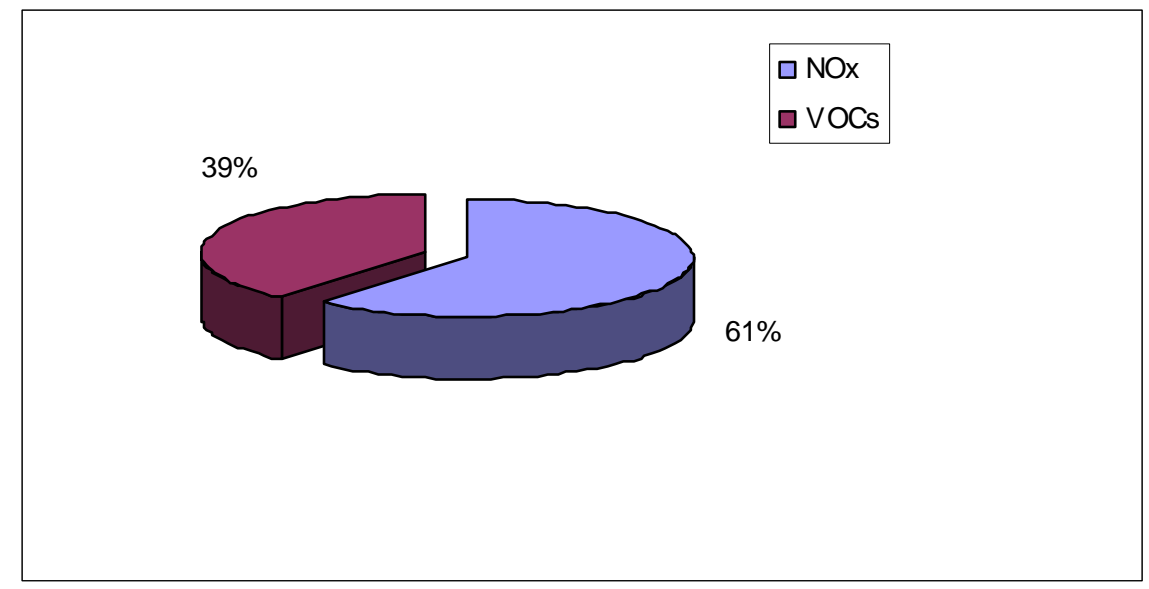

Figure 13. Percent Contribution by Substance to the Normalized value for Photochemical Smog Formation.

\begin{tabular}{|l|c|c|c|}
\hline \multicolumn{1}{|c|}{ Substance Name } & $\begin{array}{c}\text { U.S. Normalized value } \\
\text { (NOx eq/yr) }\end{array}$ & $\begin{array}{c}\text { U.S. Normalized value } \\
\text { (NOx eq/yr/capita) }\end{array}$ & $\begin{array}{c}\text { Contribution to } \\
\text { Total } \\
\text { (in \%) }\end{array}$ \\
\hline Nitrogen Oxides (NOx) & $2.06 \mathrm{E}+10$ & $7.40 \mathrm{E}+01$ & $61.00 \%$ \\
\hline VOCs & $1.32 \mathrm{E}+10$ & $4.73 \mathrm{E}+01$ & $39.00 \%$ \\
\hline Total & $\mathbf{3 . 3 8 E + 1 0}$ & $\mathbf{1 . 2 1 E + 0 2}$ & $\mathbf{1 0 0 . 0 0 \%}$ \\
\hline
\end{tabular}

Table 26. 1999 U.S. Normalized values by Substance for Photochemical Smog Formation

FOSSIL FUEL DEPLETION

\begin{tabular}{|l|c|c|c|}
\hline \multicolumn{1}{|c|}{ Fossil Fuel } & U.S. Annual Consumption & Unit & Reference \\
\hline Oil & $4.00 \mathrm{E}+13$ & MJ/year & USDOE, 2001 \\
\hline Natural Gas & $2.34 \mathrm{E}+13$ & MJ/year & USDOE, 2001 \\
\hline Hard Coal & $2.19 \mathrm{E}+13$ & MJ/year & USDOE, 2001 \\
\hline
\end{tabular}

Table 27. Fossil Fuels with U.S. Annual Consumption Estimates

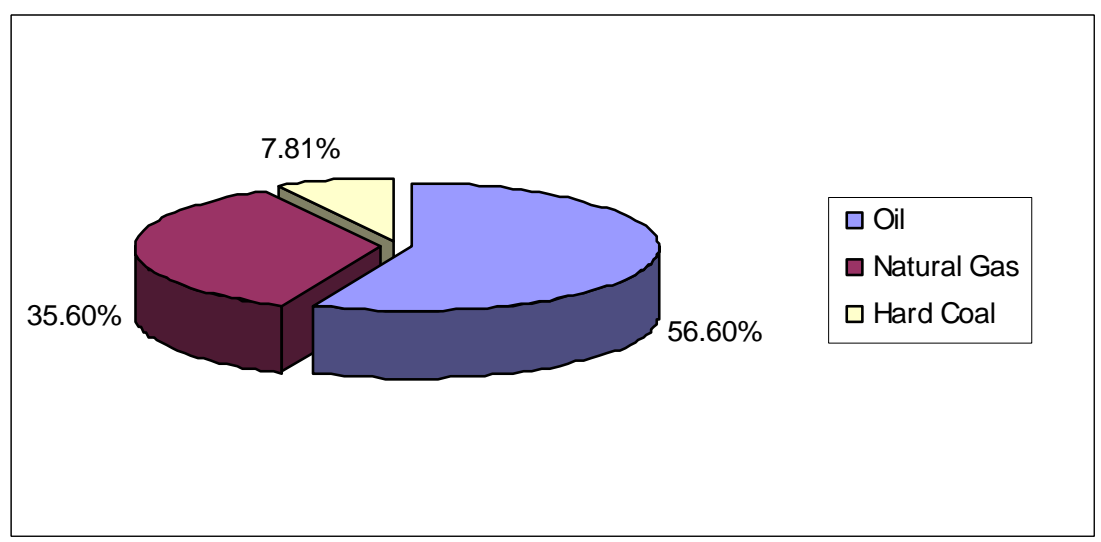

Figure 14. Percent Contribution by Fuel to the Normalized value for Fossil Fuel Use 


\begin{tabular}{|l|c|c|c|}
\hline \multicolumn{1}{|c|}{ Substance Name } & $\begin{array}{c}\text { U.S. Normalized value } \\
\text { (MJ Surplus/year) }\end{array}$ & $\begin{array}{c}\text { U.S. Normalized Value } \\
\text { (MJ Surplus/year per } \\
\text { capita) }\end{array}$ & $\begin{array}{c}\text { \% Contribution } \\
\text { to Total }\end{array}$ \\
\hline Oil & $6.45 \mathrm{E}+06$ & $2.31 \mathrm{E}-02$ & $56.60 \%$ \\
\hline Natural Gas & $4.05 \mathrm{E}+06$ & $1.45 \mathrm{E}-02$ & $35.60 \%$ \\
\hline Hard Coal & $8.89 \mathrm{E}+05$ & $3.19 \mathrm{E}-03$ & $7.81 \%$ \\
\hline Total & $\mathbf{1 . 1 4 E + 0 7}$ & $\mathbf{4 . 0 8 E}-\mathbf{0 2}$ & $\mathbf{1 0 0 . 0 0 \%}$ \\
\hline
\end{tabular}

Table 28. 1999 U.S. Normalized values by Substance for Fossil Fuel Use. 